Accelerator Division

Alternating Gradient Synchrotron Department

BROOKHAVEN NATIONAL LABORATORY

Upton, New York 11973

Accelerator Division

Technical Note

AGS/AD/Tech. Note No. 378

\title{
BUCKET PARAMETERS AND BUNCHING FACTOR FOR DOUBLE RF SYSTEM
}

\author{
Joseph M. Kats
}

August 4, 1993

\begin{abstract}
The addition of a second harmonic cavity to the main rf system can help to increase the intensity of the beam without increasing (or with reducing) instabilities. This advantage of the double rf system comes from its flexibility. The second harmonic of the double rf system has its own peak voltage, as well as phase shift relative to the first harmonic. These two parameters allow the change of the shape of the bucket and the bunch in many different ways.

In this report it will be shown how to calculate the bucket and bunch parameters for an optimized double rf system and then this will be compared to the single rf system to see how much improvement for the bunching factor and for capture efficiency can be achieved by the double rf system relative to the single rf system.
\end{abstract}


1. INTRODUCTION (Why Double RF System?) . . . . . . . 1

1.1 Instabilities and Particle Losses . . . . . . . . . . 1

1.2 The Current . . . . . . . . . . . . . . . 1

1.3 The Hamiltonian, Potential and Voltage . . . . . . . 2

1.4 Phase Density . . . . . . . . . . . . . . 3

1.5 Line Density . . . . . . . . . . . . . . 3

1.6 Connection Between the Current and the Voltage .... 4

1.7 Why Double Rf System? . . . . . . . . . . . . 5

1.8 Two Approaches to the Problem of Minimization .... 5

2. SYNCHROTRON EQUATIONS AND BASIC

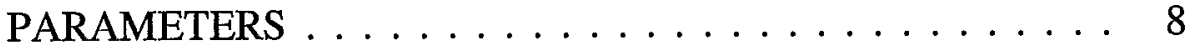

2.1 Hamiltonian Equations $\ldots \ldots \ldots \ldots \ldots \ldots$

2.2 Double Voltage ................. 9

2.3 Synchronous Particle and Acceleration Rate ...... 9

2.4 Double Potential . . . . . . . . . . . . . . . . 11

2.5 Synchronous Phase, Second Amplitude and Phase

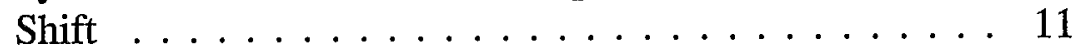

2.6 Voltage Constraints . . . . . . . . . . . . 14

3. FIXED POINTS $\ldots \ldots \ldots \ldots \ldots \ldots \ldots \ldots$

3.1 Hamiltonian Surface . . . . . . . . . . . . . . 15

3.2 Classification of Fixed Points . . . . . . . . . 16

3.3 Unstable Fixed Point . . . . . . . . . . . . . . . 19

4. COMPARISON OF SINGLE AND DOUBLE RF

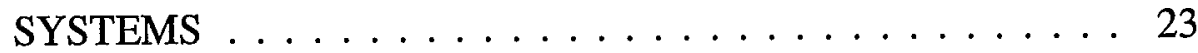

4.1 Choosing the Basis for Comparison $\ldots \ldots \ldots \ldots 23$

4.2 The Bucket and Its Height . . . . . . . . . . . . 24

4.3 Voltage Connection for the Two Systems and Data for the Power Supply . . . . . . . . . . . . . . 25

4.4 Bucket Length and Area . . . . . . . . . . . . . . 29

4.5 Synchrotron Frequency . . . . . . . . . . . . 31

4.6 Bunching Factor . . . . . . . . . . . . . 38

4.7 Capture Efficiency . . . . . . . . . . . . . . . 41

ACKNOWLEDGMENTS ... . . . . . . . . . . 42

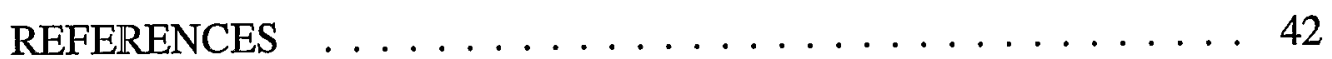




\section{INTRODUCTION (Why double rf system?)}

The purpose of this section is to show how the inhomogeneity of the current is connected to the cavity voltage. The single rf system has no tools to manipulate the voltage wave in order to reduce current inhomogeneities. In contrast, the double rf system possess degrees of freedom enough to change the shape of the voltage wave that reduces current inhomogeneities, and by doing so, increases stability and intensity of the beam.

\subsection{Instabilities and Particle Losses}

The main product of every accelerator is a particle beam (sometimes it is not a beam, but rather a radiation created by the beam as in BNL's NSLS). The main qualities of that product are energy, luminosity, and intensity.

The higher energy is important to penetrate into the higher branches of elementary particle hierarchy. Higher luminosity increases the probability of nuclear reactions per unit cross section of the collision. Higher intensity increases the probability of the same reaction per unit of time. To be specific, let us talk about intensity.

If we try to increase intensity by injecting more and more particles in the accelerator, then sooner or later we will face particle losses. There are many stages in the acceleration cycle where particles are lost. They hit hardware and residual gas in the chamber, producing various damage and dangerous radiation. There are losses due to the poor longitudinal capture, due to various transverse resonances, due to transition energy crossing, due to the complexity of extraction, and so on. Many losses can occur due to a wide class of longitudinal instabilities. Most of them manifest themselves as instabilities of the flow of charged particles, which is the electrical current circulating around the machine.

\subsection{The Current}

In the longitudinal reference frame, the current is a function of the longitudinal coordinate $\phi: I=I(\phi)$. On the other hand, according to its definition, the current is a number of charged particles passing a point $\phi$ per unit time:

$$
I(\phi)=q \frac{d N}{d t}=q \frac{d N}{d \phi} \cdot \frac{d \phi}{d t}=\frac{q \omega}{2 \pi} \cdot \frac{d N}{d \phi}=\text { const } \cdot \frac{d N}{d \phi}
$$

where $q$ is the particle charge, $\omega$ is the revolution frequency, $N=N(\phi)$ is a number of particles corresponding to point $\phi$, and $d N=N(\phi+d \phi)-N(\phi)$. 
A vast majority of longitudinal instabilities occurs due to current inhomogeneity $d I / d \phi$, when its relative value $d I / d \phi / I$ exceeds some threshold. To evaluate the area of current stability and instability means to evaluate $d I / d \phi$. This inhomogeneity can either grow producing instability or it can decay providing a stable evolution of particle flow.

\subsection{The Hamiltonian, Potential and Voltage}

In classical mechanics, a Hamiltonian is a sum of kinetic energy of the moving particle and potential energy of interaction between the particle and a field. In accelerator mechanics, a Hamiltonian is a sum of potential energy and appropriate function of a particle's energy. As we will see later in Section 2, the Hamiltonian can be written as

$$
H(\phi, W)=-m \cdot \frac{W^{2}}{2}-U(\phi),
$$

where $U(\phi)$ is a potential of the cavity's electrical field, $W$ is the particle's normalized energy, and $m$ is a coefficient combined from a set of accelerator parameters ( $m$ is not a mass).

A potential $U$ is a scalar field, whose gradient is a force, which is a voltage (more precisely: a difference between applied voltage and synchronous voltage):

$$
\frac{d U}{d \phi}=V(\phi)-V_{s}
$$

The voltage $V$ is a product of voltage amplitude $V_{o}=V_{o}(t)$, sometimes called a peak-voltage, and a voltage wave $v=v(\phi)=v(\phi, t)$ :

$$
V(\phi)=V_{o}(t) \cdot v(\phi) \text {. }
$$

For example, for a single rf system, the voltage wave is $v(\phi)=\operatorname{Sin}(\phi)$. Similarly to Eq. (4), a synchronous voltage $V_{s}$ is a product

$$
V_{s} \equiv V_{s}(t)=V_{o} \cdot v\left(\phi_{s}\right) \equiv V_{o} \cdot v_{s},
$$

where $\phi_{s}$ is a synchronous phase.

In this section, the Hamiltonian will provide a connection between a current $I(\phi)$ and a potential $U(\phi)$. The connection will be established by use of phase density $\rho$. 


\subsection{Phase Density}

In a phase plane $(\phi, W)$, the number of particles corresponding to the point $(\phi, W)$ is a function $N=N(\phi, W)$. A local or phase density is

$$
\rho(\phi, W)=\frac{d^{2} N}{d \phi d W}
$$

Particles' motion and the evolution of their phase density depend on the state of the accelerator controlled by magnetic field $B$, cavity voltage $V$, and frequency, $f$. If all three $B, V, f$ are changing with time slowly enough, we say there is adiabatic conditions in a phase space.

The most important property of the local density $\rho$ is that under adiabatic conditions, the local density is reduced to the function of one variable, which is the Hamiltonian $H$ :

$$
\rho(\phi, W)=\rho(H)
$$

\subsection{Line Density}

Experimentally, we cannot directly observe the phase density distribution. What we can see is the so-called line density $\lambda$ or "mountain range", which is an integral of phase density, $\rho$, over all particles with the given phase angle $\phi$ :

$$
\lambda(\phi)=\int \rho(W, \phi) d W=\int \frac{d^{2} N}{d \phi d W} d W=\frac{d N}{d \phi},
$$

which is to within a constant factor is the same as a current (1):

$$
I(\phi)=\text { const } \cdot \lambda(\phi) .
$$

Under adiabatic conditions, we can combine (7), (8), and (9) to get

$$
I(\phi)=\text { const } \cdot \int \rho(H) d W .
$$




\subsection{Connection Between the Current and the Voltage}

To evaluate the current inhomogeneity, we have to find

$$
\frac{d I}{d \phi}=\text { const } \cdot \int \frac{\partial \rho}{\partial H} \cdot \frac{\partial H}{\partial \phi} d W
$$

As $\frac{\partial H}{\partial \phi}=-\frac{d U}{d \phi}$ does not depend on $W$, one can rewrite Eq. (11) as

$$
\frac{d I}{d \phi}=\frac{d U}{d \phi} \cdot F(\phi)
$$

where $F(\phi)$ is some function of $\phi$ :

$$
F(\phi)=\text { const } \cdot \int \frac{\partial \rho}{\partial H} d W
$$

The idea of a second harmonic cavity as a cure for longitudinal instabilities was brought up precisely because of the last formula. Indeed, we will see later in Section 2 that the parameters for the double rf system can be chosen in such a way that within the bucket there will be a significantly wide area around the center where the Hamiltonian does not depend on the phase angle $\phi$. For such an area, density $\rho$ will also be a function of $W$ only. Consequently the function $F(\phi)$ will degenerate to the simple constant.

Leaving this function $F$ for a future study, right now we can say, that because of Equations (3), (8), and (11), an inhomogeneity of the current (as well as of the line density) is proportional to the voltage

$$
\frac{d I}{d \phi}=V_{o} \cdot\left[v(\phi)-v_{s}\right] \cdot F(\phi) .
$$
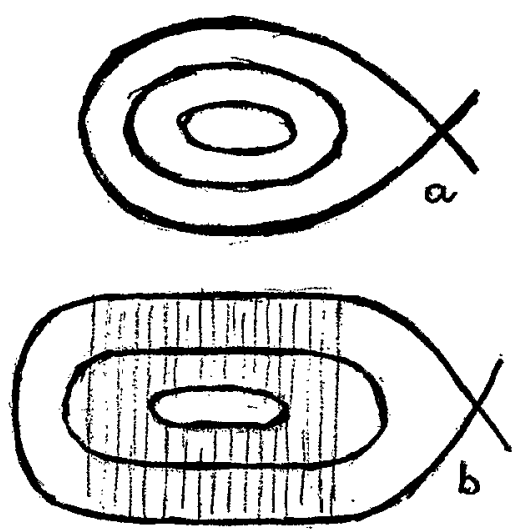

Figure 1. Particle's trajectories for the single (a) and double (b) rf system. The shaded area is where the Hamiltonian's dependence on phase $\phi$ is very weak.

Thus, in order to reduce current inhomogeneity, we have to minimize the deviation of the voltage wave $v(\phi)$ from its synchronous value $v_{\mathrm{s}}$. 


\subsection{Why Double RF System?}

Multiple rf systems with second, third, fourth, and higher harmonic cavities were subjects of experimental and theoretical studies for a number of years [1-7]. This report differs from the given references in the exact statement of the problem as to how the multiple system should be optimized. Also for the first time, in this report we provide an exact solution for an optimal double rf system.

If we deal with just the main (single) rf system, then we have

$$
V(\phi)-V_{s}=V_{o}\left(\operatorname{Sin} \phi-\operatorname{Sin} \phi_{s}\right) \text {. }
$$

In this case, the only way to minimize $V-V_{s}$ is to reduce the voltage amplitude (peak) $V_{o}$. However, $V_{o}$ is not a free parameter. It has a lower limit which insures the existence of the longitudinal bucket. It has lower and upper constraints during injection, capture, acceleration, transition, and extraction.

Thus, the single rf system cannot actively influence particle distribution within the bunch. The single rf system is a passive vehicle driving particles toward the higher energy. Unfortunately, the driver is blind. That is why we need a second driver, who will watch many turns, lines and signs along the road. This driver is an additional rf system, particularly the second harmonic cavity.

When we have a double rf system, then two new joysticks, two new parameters $r$ and $\delta$ are at our disposal

$$
V(\phi)=V_{o}[\operatorname{Sin} \phi+r \cdot \operatorname{Sin} 2(\phi-\delta)] .
$$

With the given $V_{o}$, the second amplitude $\mathrm{r}$ (actually it is $r V_{o}$ ) and the phase shift $\delta$ are two free parameters. By changing $r$ and $\delta$, we can change $V(\phi)$ throughout the whole region $\phi_{\ell} \leq \phi \leq \phi_{\mathrm{r}}$ occupied by bunch, where $\phi_{\iota}$ and $\phi_{\mathrm{r}}$ are left and right phase boundaries of the bunch. Now we can discuss the minimization of $d I / d \phi$ using $r, \delta$ without touching $V_{o}$.

\subsection{Two Approaches to the Problem of Minimization}

There are many ways to minimize $V(\phi)-V_{s}$. All these ways depend on the segment $\phi_{\mathrm{a}} \leq \phi \leq \phi_{\mathrm{b}}$, where minimization is required and on the nature of a functional to be minimized. Obviously, underlying segment should be within a phase region of the bunch:

$$
\phi_{\ell} \leq \phi_{a} \leq \phi \leq \phi_{b} \leq \phi_{r}
$$




\subsubsection{Integral Approach}

One of the natural looking functions is a mean deviation. The problem is to find such parameters $\phi_{\mathrm{s}}, r, \delta$ that satisfy

$$
\underset{\phi_{s}, r, \delta}{\operatorname{minimum}} \int_{\phi_{a}}^{\phi_{b}}\left[V(\phi)-V_{s}\right]^{2} d \phi
$$

for the given $\phi_{\mathrm{a}}, \phi_{\mathrm{b}}$, in particular, for $\phi_{\mathrm{a}}=\phi_{\ell}, \phi_{\mathrm{b}}=\phi_{\mathrm{r}}$. This approach can lead to the bucket which has two stable fixed points (see Figure 2). This in turn, can create inhomogeneity in line density $\lambda$ and in current $I$.

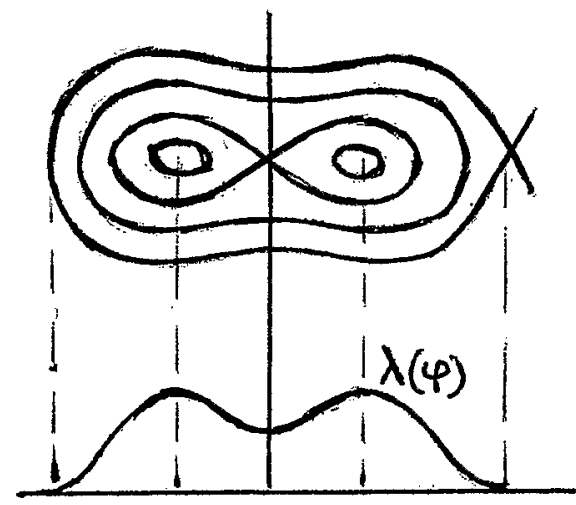

Figure 2. A bucket with two fixed points and corresponding line density with two peaks.

\subsubsection{Local Approach}

Another approach to the minimization of $V(\phi)-V_{s}$ is coming from the local properties of voltage wave $V=V(\phi)$ at the synchronous phase $\phi=\phi_{s}$. As $V\left(\phi_{\mathrm{s}}\right)-V_{\mathrm{s}}=0$, it would be nice to have a function

$$
\Delta V=\Delta V(\phi)=V(\phi)-V_{s}
$$

in a form of a power:

$$
\Delta V(\phi)=\text { const } \cdot\left(\phi-\phi_{s}\right)^{n},
$$

which rectifies (flattens) a voltage $V(\phi)$ around point $\phi=\phi_{\mathrm{s}}$ as it is shown in Figure 3. The larger $n$ is, the wider the interval, where $V(\phi)$ is (almost) equal to the constant $V_{s}$. In other words, the larger $n$ is, the better the minimization is. 


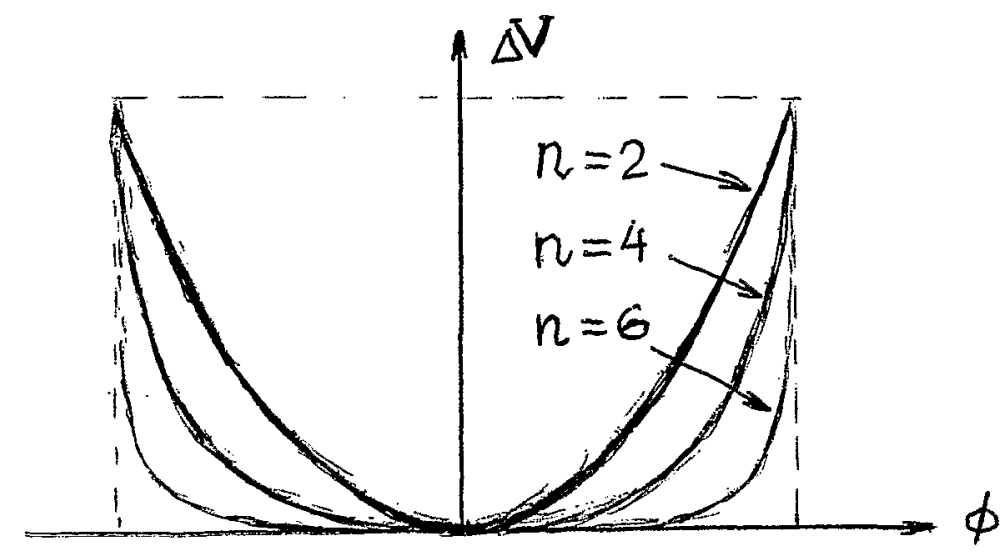

Figure 3. Minimization with the power function.

To find a representation like (20), we first Taylor expand the function (19) around $\phi=\phi_{\mathrm{s}}, V_{\mathrm{s}}=V\left(\phi_{\mathrm{s}}\right)$ :

$$
V(\phi)=\sum_{k=0}^{\infty} \frac{V_{s}^{(k)}}{k !}\left(\phi-\phi_{s}\right)^{k}
$$

and then we seek the conditions to zero the first $\mathrm{n}-1$ Taylor terms:

$$
\left.\frac{d^{m}\left[V(\phi)-V_{s}\right]}{d \phi^{m}}\right|_{\phi=\phi_{s}}=0, \quad m=1,2, \ldots n-1 .
$$

If we satisfy such conditions, then the voltage wave is a power type function:

$$
V(\phi)-V_{s}=O\left[\left(\phi-\phi_{s}\right)^{n}\right]
$$

The larger is $n$, the wider is the interval where the left-hand side of (23) is as minimal as possible.

As we have only three free parameters $\phi_{s}, r, \delta$, we have to restrict ourselves to three equations in the system (22). If we would have in addition to the second harmonic cavity, a third harmonic cavity as well, then there would be five parameters $\phi_{s}, \mathrm{r}_{2}, \delta_{2}, \mathrm{r}_{3}, \delta_{3}$ with indices 2,3 referring to the second and third harmonic. Having this, we would be able to raise the order $m$ in (22) to have 5 equations for 5 parameters and to flatten $V(\phi)$ even more. But first, we have to explore how far we can go with the second harmonic. 


\section{SYNCHROTRON EQUATIONS AND BASIC PARAMETERS}

\subsection{Hamiltonian Equations}

In the longitudinal phase plane $(\phi, W)$, the synchrotron motion of charged particles is governed by Hamiltonian equations

$$
\left\{\begin{array}{l}
-\frac{\partial H}{\partial \phi}=\frac{d W}{d t}=\frac{q}{2 \pi}\left[V(\phi, t)-V_{s}\right]=\frac{q}{2 \pi} \cdot \frac{d U}{d \phi} \\
\frac{\partial H}{\partial W}=\frac{d \phi}{d t}=-m W
\end{array}\right.
$$

which can be derived from (or reduced to) the Hamiltonian

$$
H(\phi, W)=-m \frac{W^{2}}{2}-\frac{q}{2 \pi} U\left(\phi, \phi_{s}\right),
$$

where

$$
\left\{\begin{aligned}
W & =\left(E-E_{s}\right) / \omega_{s} & & m=\omega_{s}^{2} h \eta / \beta_{s}^{2} E_{s}, \\
h \omega_{s} & =2 \pi f, & & =\beta_{t}^{2}-\beta_{s}^{2}, \\
\gamma & =E / E_{r}, & & \beta^{2}=1-1 / \gamma^{2}, \\
q & =Q e / A, & & E_{r}=I_{r} .
\end{aligned}\right.
$$

Here $E, \phi$ are the particle's energy (per nucleon) and phase. The subscripts $\mathbf{r}$, $\mathrm{s}$, and $\mathrm{t}$ refer to the rest, synchronous, and transition energy. Please note the use of notations with $\eta_{s}>0, m>0$ below transition energy (because the Hamiltonian or at least its kinetic part is a sign-definitive function; to show it explicitly, I choose the listed notations).

For a synchronous particle, the revolution frequency $\omega_{\mathrm{s}}$ is locked to the main $\mathrm{rf}$ frequency $f$ via the harmonic number $h$. The other notations are as follows. $A$ is the atomic mass number, $I_{\mathrm{r}}$ is the ionic rest energy per nucleon, $\mathrm{Q}$ is the ion's charge state (number of stripped electrons from the atom), $e$ is the proton charge, $V$ is a voltage function, $U$ is a potential field:

$$
\frac{d U}{d \phi}=V(\phi, t)-V_{s} .
$$




\subsection{Double Voltage}

Along with the main rf system, we will consider a second harmonic system, such that the effective voltage can be described as

$$
\begin{gathered}
V(\phi) \equiv V(\phi, t)=V_{o}(t) \cdot v(\phi, t) \equiv V_{o} v, \\
v(\phi) \equiv v(\phi, t)=\operatorname{Sin} \phi+r \operatorname{Sin} 2(\phi-\delta),
\end{gathered}
$$

where $V_{0} \geq 0$ is the main (first) amplitude, $r=r(t)$ is a second amplitude (as a fraction of $\left.V_{\mathrm{o}}\right), \delta=\delta(\mathrm{t})$ is a phase shift of the second harmonic with respect to the first harmonic.

We call $V$ voltage, $v$ voltage wave. Voltage is a periodic function whose mean value is zero:

$$
V(\phi+2 \pi)=V(\phi), \quad \int_{\phi}^{\phi+2 \pi} V(x) d x=0 .
$$

\subsection{Synchronous Particle and Acceleration Rate}

A synchronous particle $\left(\phi_{s}, E_{s}\right)$ provides a reference frame for all others (asynchronous) particles $(\phi, E)$ or $(\phi, W)$. In fact, a refrence frame is provided not by the physical synchronous particle, but rather by the parameters of the machine to which a corresponding synchronous particle would match its phase $\phi_{\mathrm{s}}$ and energy $E_{s}$. [8]

Let us find a function $E_{\mathrm{s}}=E_{\mathrm{s}}\left(\phi_{\mathrm{s}}\right)$, as well as a connection between a synchronous particle $\left(\phi_{s}, E_{s}\right)$ and machine parameters. First, we write a longitudinal dynamic equation for the synchronous particle

$$
\frac{d E_{s}}{d t}=\frac{q \omega_{s}}{2 \pi} \cdot V_{s}
$$

where the synchronous voltage $V_{s}$ is

$$
V_{s}=V\left(\phi_{s}, t\right)=V_{o}(t) \cdot v\left(\phi_{s}\right) \text {. }
$$

Thus, Equation (7) shows how the rate of change $\dot{E}_{s}$ is connected with synchronous phase $\phi_{s}$. Now we will connect the same $\dot{E}_{s}$ with the magnetic field and other parameters. 
If a particle stays on the machine's central orbit of radius $R$, then the particle momenta $p_{\mathrm{s}}$ satisfies the Lorentz equation

$$
p_{s}=q \rho B
$$

where $q$ is charge, $\rho=$ const. is a curvature radius of the main dipole magnet, whose field is $B=B(t)$.

The rate of change of momenta is $\dot{p}_{s}=q \rho \dot{B}$, while the rate of change of the energy is ( $v=\omega R$ is the particle's linear velocity)

$$
\dot{E}_{s}=\frac{d E_{s}}{d p_{s}} \cdot \dot{p}_{s}=v \cdot \dot{p}_{s}=\omega_{s} R \cdot q \rho \dot{B}
$$

By equating (7) and (10), we get

$$
V_{s}=2 \pi R \rho \dot{B},
$$

or

$$
v_{s} \equiv v\left(\phi_{s}\right)=2 \pi R \rho \dot{B} / V_{o}=V_{s} / V_{o},
$$

and for the double system (first harmonic plus the second harmonic)

$$
v_{s}=\operatorname{Sin} \phi_{s}+r \cdot \operatorname{Sin} 2\left(\phi_{s}-\delta\right) \text {. }
$$

We call $v_{\mathrm{s}}$ a (normalized) rate of acceleration.

The evolution of synchronous energy $E_{s}=E_{s}(t)$ characterizes an energy of the evolving bunch as a whole: if $v_{s}>0$, then $\dot{E}_{s}>0$ and the bunch is accelerating, and vice versa.

In longitudinal phase space $(\phi, W)$, we prescribe to every particle its energy $W=\left(E-E_{s}\right) / \omega_{s}$ measured relative to the synchronous energy $E_{\mathrm{s}}$ and normalized by $\omega_{\mathrm{s}}$. For this reason, the force acting upon the particle is voltage $V-V_{s}$ measured relative to its synchronous value $V_{s}$. There is a slight ambiguity when we use a word "voltage" meaning sometimes $V$ and other times $V-V_{s}$. I hope the reader recognizes from the context "who is who". 


\subsection{Double Potential}

Similar to the cavity voltage, the potential can be split into two factors-amplitude and wave:

$$
\begin{gathered}
U\left(\phi, \phi_{s}\right)=V_{o}\left[u_{s}-u(\phi)\right], \\
u(\phi)=\operatorname{Cos} \phi+\frac{r}{2} \operatorname{Cos} 2(\phi-\delta)+\phi v_{s}, \quad u_{s}=u\left(\phi_{s}\right) .
\end{gathered}
$$

We call $u=u(\phi)$ a potential wave. Potential as well as Hamiltonian is determined only to within an additive constant which has no effect on the equation of motion. By denoting a potential as $U\left(\phi, \phi_{s}\right)$, we show explicitly that the additive constant is determined by the phase angle $\phi_{s}$. Choosing that constant equal to $V_{\mathrm{o}} u_{\mathrm{s}}$, we make a potential equal to zero when $\phi=\phi_{\mathrm{s}}$ :

$$
U\left(\phi_{s}, \phi_{s}\right)=0 \text {. }
$$

It follows from (4), (5), and (14) that the relationship between the potential wave and the voltage wave can be established without involving an amplitude $V_{0}$ :

$$
\frac{d u}{d \phi}=v(\phi)-v_{s} \text {. }
$$

\subsection{Synchronous Phase, Second Amplitude and Phase Shift}

To find three unknowns, $\phi_{\mathrm{s}}, r, \delta$, we have to use three equations $(\mathrm{n}=3)$ of the system (1.22). Using prime ' $\equiv d / d \phi$, we write:

$$
\left\{\begin{aligned}
v\left(\phi_{s}\right) & =v_{s} \\
v^{\prime}\left(\phi_{s}\right) & =0 \\
v^{\prime}\left(\phi_{s}(\right. & =0
\end{aligned}\right.
$$

or

$$
\left\{\begin{array}{l}
f_{1}\left(\phi_{s}, r, \delta\right)=\operatorname{Sin} \phi_{s}+r \operatorname{Sin} 2\left(\phi_{s}-\delta\right)-v_{s}=0 \\
f_{2}\left(\phi_{s}, r, \delta\right)=\operatorname{Cos} \phi_{s}+2 r \operatorname{Cos} 2\left(\phi_{s}-\delta\right)=0 \\
f_{3}\left(\phi_{s}, r, \delta\right)=\operatorname{Sin} \phi_{s}+4 r \operatorname{Sin} 2\left(\phi_{s}-\delta\right)=0
\end{array}\right.
$$

Before we start to solve this system, we will check its self-consistency by evaluating the Jacobian of the three functions $f_{1}, f_{2}, f_{3}$ of the three variables $\phi_{s}$, $r, \delta$. If the Jacobian is non-zero, then $f_{1}, f_{2}, f_{3}$ are functionally independent, and Equation (19) has at least one set of roots. 


$$
\begin{aligned}
J & =\operatorname{det}\left(\frac{\partial f_{i}}{\partial x_{k}}\right)= \\
& =\left|\begin{array}{ccc}
\operatorname{Cos} \phi_{s}+2 r \operatorname{Cos} 2\left(\phi_{s}-\delta\right) & \operatorname{Sin} 2\left(\phi_{s}-\delta\right) & -2 r \operatorname{Cos} 2\left(\phi_{s}-\delta\right) \\
-\operatorname{Sin} \phi_{s}-4 r \operatorname{Sin} 2\left(\phi_{s}-\delta\right) & 2 \operatorname{Cos} 2\left(\phi_{s}-\delta\right) & 4 r \operatorname{Sin} 2\left(\phi_{s}-\delta\right) \\
\operatorname{Cos} \phi_{s}+8 r \operatorname{Cos} 2\left(\phi_{s}-\delta\right) & 4 \operatorname{Sin} 2\left(\phi_{s}-\delta\right) & -8 r \operatorname{Cos} 2\left(\phi_{s}-\delta\right)
\end{array}\right|= \\
& =-12 r \operatorname{Cos} \phi_{s} .
\end{aligned}
$$

Thus, as soon as $r \operatorname{Cos} \phi_{s} \neq 0$, the system (19) determines at least one set of roots. The roots can be found as follows. From the first and second equations of (19), one gets:

$$
\begin{aligned}
\operatorname{Sin} \phi_{s} & =\frac{4}{3} v_{s} \equiv s, \\
\phi_{s} & =\arcsin s .
\end{aligned}
$$

Then, after dividing the third equation by the second, one gets

$$
2 \tan 2\left(\phi_{s}-\delta\right)=\tan \phi_{s}
$$

or

$$
\delta=\phi_{s}-\frac{1}{2} \arctan \frac{\tan \phi_{s}}{2}
$$

Finally, by raising to the second power and adding the second and third, we have

$$
r^{2}=\frac{1}{4}\left[1-\frac{3}{4} s^{2}\right]=\frac{1}{4}-\frac{v_{s}^{2}}{3} .
$$

The root of the system (1) is a single set of three numbers $\phi_{s}, r\left(\phi_{s}\right), \delta\left(\phi_{s}\right)$. Let $\phi_{\mathrm{p}}=\pi-\phi_{\mathrm{s}}$. If $\left.\left[\phi_{\mathrm{s}}, \mathrm{r} \phi_{\mathrm{s}}\right), \delta\left(\phi_{\mathrm{s}}\right)\right]$ is a root of (1) then $\left.\left[\phi_{\mathrm{p}}, r \phi_{\mathrm{p}}\right), \delta\left(\phi_{\mathrm{p}}\right)\right]$ is not a root, although $\phi_{\mathrm{p}}$ does satisfy (21). This is in contrast with a single rf system where $\phi$ and $\phi_{\mathrm{u}}=\pi-\phi_{\mathrm{s}}$ both satisfy $\operatorname{Sin} \phi=v_{\mathrm{s}}$.

System (19) is invariant under each of the following transformations

$$
\begin{gathered}
\phi \Rightarrow \phi \pm 2 \pi, \\
\delta \Rightarrow \delta \pm \pi,
\end{gathered}
$$




$$
\left\{\begin{array}{l}
r \Rightarrow-r \\
\delta \Rightarrow \pm \frac{\pi}{2}
\end{array}\right.
$$

If

$$
0 \leq \phi_{s} \leq \frac{\pi}{2}
$$

then according to Eq. (24) the phase shift should satisfy

$$
0 \leq \delta \leq \phi_{s} \text {. }
$$

Then from the last equation of system (19), it follows that

$$
r \leq 0 \text {. }
$$

Thus, the solution of system (19) is

$$
\left\{\begin{array}{ll}
\phi_{s}=\arcsin s, & 0 \leq \phi_{s} \leq \frac{\pi}{2}, \\
\delta=\phi_{s}-\frac{1}{2} \operatorname{arctg} \frac{\operatorname{tg} \phi_{s}}{2}, & 0 \leq \delta \leq \frac{\pi}{4}, \\
r=-\sqrt{\frac{1}{4}\left(1-\frac{3}{4} s^{2}\right)}=-\sqrt{\frac{1}{4}-\frac{v_{s}^{2}}{3}}, & 0 \leq|r| \leq \frac{1}{2} .
\end{array} .\right.
$$

Figure 4 shows the functions $\delta=\delta\left(\phi_{s}\right)$ and $r=r\left(\phi_{s}\right)$.
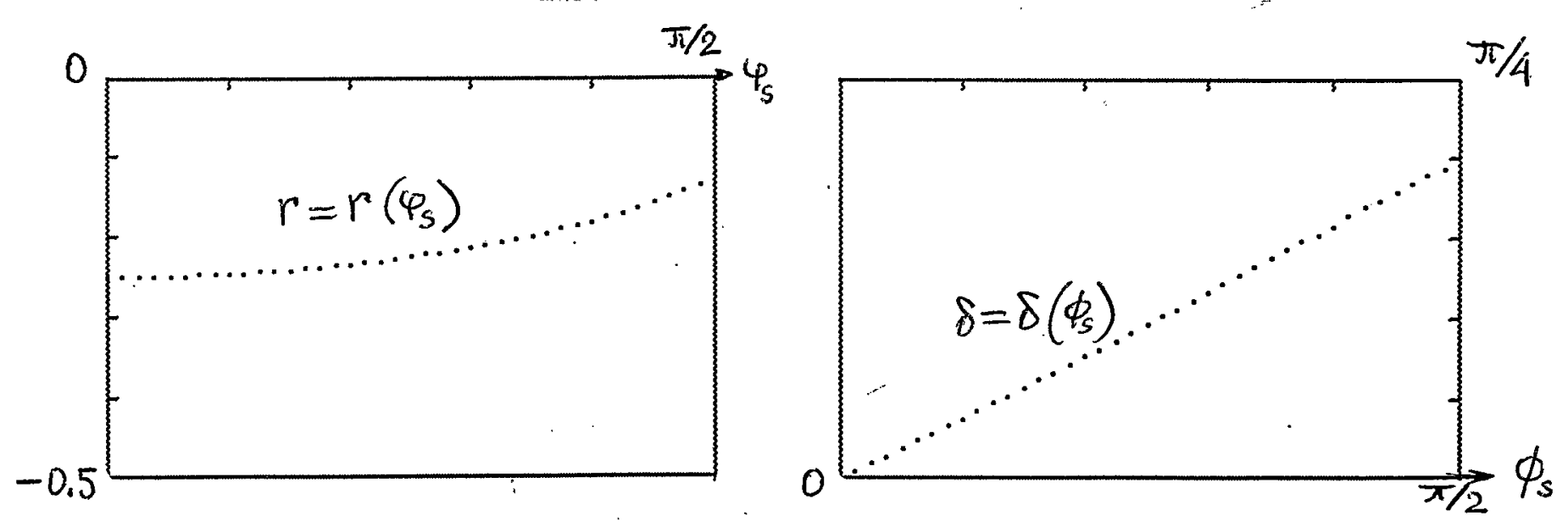

Figure 4. Second voltage $r$ and phase shift $\delta$ as a function of the synchronous angle $\phi_{\mathrm{s}}$. 


\subsection{Voltage Constraints}

In an accelerating regime $v_{s}>0$, it follows from Eq. (21) that

$$
0<v_{s} \leq \frac{3}{4} \text {. }
$$

Applying this to Eq. (12), we find

$$
V_{o}(t) \geq \frac{4}{3} \cdot V_{s}=\frac{4}{3} \cdot 2 \pi R \rho \dot{B}(t) .
$$

This is the first voltage constraint. If at any time the amplitude $V_{0}$ falls lower than the right-hand side of Eq. (34), then the bucket will cease to exist.

From (32) and (33), it follows that

$$
\frac{1}{4} \leq|r| \leq \frac{1}{2} \text {. }
$$

This is the second voltage constraint. 


\section{FIXED POINTS}

\subsection{Hamiltonian Surface}

The Hamiltonian function $H=H(\phi, W)$, being a function of two variables, is a surface (Figure 5) in three-dimensional space $(\phi, W, H)$ :

$$
H(\phi, W)=-m \frac{W^{2}}{2}-\frac{q}{2 \pi} U\left(\phi, \phi_{s}\right) .
$$

Any plane $\mathrm{H}=$ const., perpendicular to the $\mathrm{H}$-axis, intersects the Hamiltonian surface along the curve whose projection into the phase plane $(\phi, W)$ is the particle's trajectory. Trajectories can be divided into two classes--closed and open. The separatrix is a self-crossing curve which divides these two classes. The area inside of the closed part of the separatrix, the bucket, covers all the closed trajectories.

There can only be open trajectories outside of the bucket. The Hamiltonian surface possesses stationary points--local minimae and maximae--whose projection into the phase plane are fixed points.

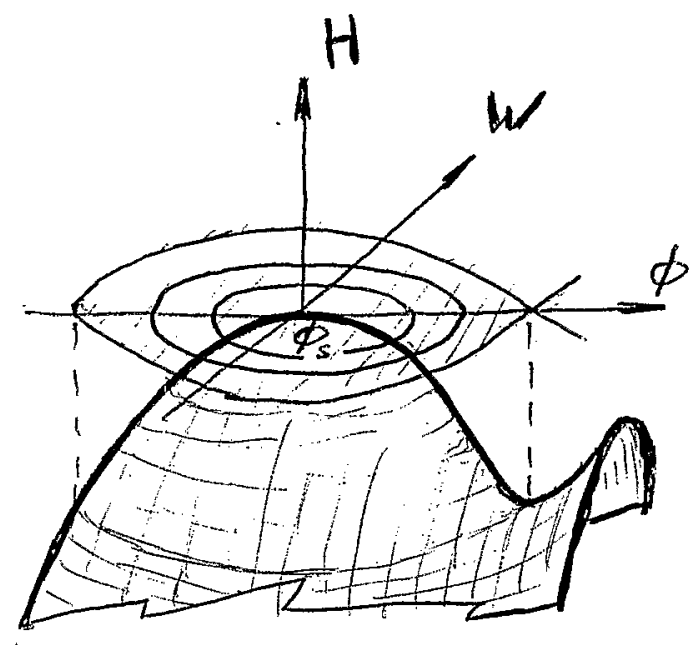

Single RF

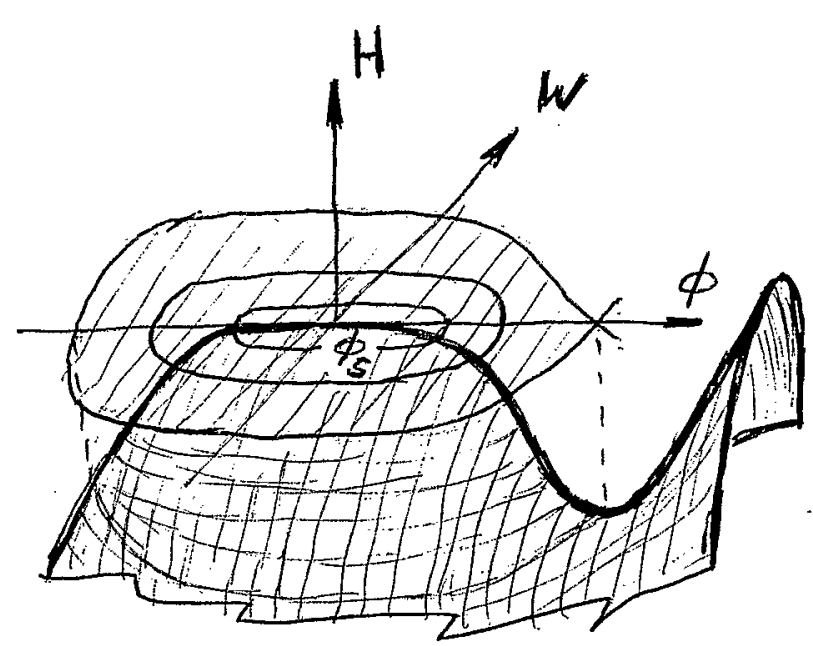

Double RF

Figure 5. Hamiltonian surface. 
All the stationary points satisfy the system of equations

$$
\left\{\begin{array}{l}
\frac{\partial H}{\partial \phi}=-\frac{q}{2 \pi} \cdot \frac{d U}{d \phi}=0 \\
\frac{\partial H}{\partial W}=-m W=0
\end{array}\right.
$$

From the second equation, it is obvious that all fixed points are lying along the $\phi$-axis:

$$
W=0
$$

and from the first equation (2), it is obvious that the position of all fixed points are determined by the potential

$$
\frac{d U}{d \phi}=0
$$

which due to (2.14) can be written in terms of potential wave

$$
\frac{d u}{d \phi}=0 \text {. }
$$

\subsection{Classification of Fixed Points}

All the fixed points are classified according to the topological structure of the particles' trajectories in the vicinity of the fixed points in question. There are stable and unstable fixed points. In the vicinity of the stable fixed points, any trajectory is closed. In the vicinity of the unstable fixed point, any trajectory is open.

To examine a trajectory in the vicinity of any fixed point $\left(\phi_{0}, 0\right)$, we select that trajectory by it phase amplitude $\Delta \phi$. The phase amplitude determines a point $\phi=\phi_{o}+\Delta \phi$, where the trajectory crosses the $\phi$-axis. The phase amplitude also determines a value of the Hamiltonian pertaining to the trajectory:

$$
H\left(\phi_{o}+\Delta \phi, 0\right)=-\frac{q}{2 \pi} \cdot U\left(\phi_{o}+\Delta \phi, \phi_{s}\right) .
$$

Let $\phi=\phi_{o}+x$ and $\mathrm{W}$ be the coordinates of a particle moving along the trajectory under consideration and let

$$
|x| \leq|\Delta \phi| \ll 1 .
$$


We substitute in the left-hand side of the Hamiltonian (1) its constant value from (6), while on the right-hand side of (1) we put the trajectory coordinates

$$
-\frac{q}{2 \pi} \cdot U\left(\phi_{o}+\Delta \phi, \phi_{s}\right)=-m \frac{W^{2}}{2}-\frac{q}{2 \pi} U\left(\dot{\phi}_{o}+x, \phi_{s}\right),
$$

where $x, W$ are variables, $\phi_{o}, \phi_{s}, \Delta \phi$ are constants. Thus Eq. (8) is the trajectory equation in variables $x, W$. Due to $(2.14)$ we can rewrite (8) as

$$
u\left(\phi_{o}+\Delta \phi\right)=-\frac{\pi m}{q V_{o}} \cdot W^{2}+u\left(\phi_{o}+x\right)
$$

After a Taylor expansion of $u$ in both sides of (9), it becomes

$$
\begin{gathered}
u_{1} \Delta \phi+u_{2} \Delta \phi^{2} / 2 !+u_{3} \Delta \phi^{3} / 3 !+u_{4} \Delta \phi^{4} / 4 !+\ldots=-\frac{\pi m}{q V_{o}} \cdot W^{2}+ \\
u_{1} x+u_{2} x^{2} / 2 !+u_{3} x^{3} / 3 !+u_{4} x^{4} / 4 !+\ldots
\end{gathered}
$$

where $u_{i}=d^{i} u\left(\phi_{o}\right) / d \phi^{i}, i=1,2, \ldots$. As $x$ and $\Delta \phi$ are both infinitely small, it is enough to retain only non-zero terms of the lowest order in both series of (10). According to (3.5), every fixed point $\phi_{\mathrm{o}}$ satisfies

$$
u_{1}=0 \text {. }
$$

Higher order derivatives of the potential wave u depend on the type of $\mathrm{rf}$ system. For the single rf system, we have $\left(\phi_{\mathrm{o}}=\phi_{\mathrm{s}}\right.$ is a fixed point):

$$
\left\{\begin{aligned}
u(\phi) & =\operatorname{Cos} \phi+\phi v_{s}, \\
u_{1} & =d u\left(\phi_{s}\right) / d \phi=-\operatorname{Sin} \phi_{s}+v_{s}=0, \\
u_{2} & =d^{2} u\left(\phi_{s}\right) / d \phi^{2}=-\operatorname{Cos} \phi_{s}=-\sqrt{1-v_{s}^{2}}<0 .
\end{aligned}\right.
$$

In this case (10) can be written as

$$
u_{2} \Delta \phi^{2} / 2 !=-\frac{\pi m}{q V_{o}} \cdot W^{2}+u_{2} x^{2} / 2 !
$$


Because $u_{2}<0$ has the same sign as a "kinetic" term, Equation (12) is just an ellipse equation

$$
x^{2} / A^{2}+W^{2} / B^{2}=1 .
$$

This is a closed curve around a fixed point $\left(\phi_{s}, 0\right)$, which qualifies it as a stable fixed point.

For a single rf system, there is another fixed point $\phi_{u}=\pi-\phi_{s}$, which satisfies the conditions

$$
\left\{\begin{array}{l}
u_{1}=d u\left(\phi_{u}\right) / d \phi=-\operatorname{Sin} \phi_{u}+v_{s}=0 \\
u_{2}=d^{2} u\left(\phi_{u}\right) / d \phi^{2}=+\operatorname{Cos} \phi_{u}=+\sqrt{1-v_{s}^{2}}>0 .
\end{array}\right.
$$

In this case, $u_{2}>0$ has a sign opposite to the sign of the "kinetic" term, which makes (12) a hyperbolic equation

$$
x^{2} / A^{2}-W^{2} / B^{2}=1 .
$$

This is an open trajectory in the nearest vicinity of the fixed point $\left(\phi_{u}, 0\right)$, and for that reason, the fixed point is called unstable.

For a double rf system, the potential wave is

$$
u(\phi)=\operatorname{Cos} \phi+\frac{r}{2} \operatorname{Cos} 2(\phi-\delta)+\phi v_{s}
$$

and according to (2.32), there is a fixed point $\phi_{\mathrm{s}}$, which along with $r$ and $\delta$ satisfy (2.18). Our problem now is to examine whether that fixed point $\left(\phi_{s}, 0\right)$ is stable or not. Due to (2.17), we can rewrite (2.18) as

$$
u_{1}=u_{2}=u_{3}=0
$$

and from (16) we can find

$$
u_{4}=\operatorname{Cos} \phi_{s}+8 r \operatorname{Cos} 2\left(\phi_{s}-\delta\right)
$$

which can be simplified with the help of the second equation of (2.19) and (2.21):

$$
u_{4}=-3 \operatorname{Cos} \phi_{s}=-3 \sqrt{1-s^{2}}<0 \text {. }
$$


To analyze a particle's trajectory (9) in the vicinity of the fixed point $\phi_{0}=\phi_{s}$, we apply (17) and (19) to the series in (10) and we get

$$
u_{4} \Delta \phi^{4} / 4 !=-\frac{\pi m}{q V_{o}} \cdot W^{2}+u_{4} x^{4} / 4 !
$$

Because $u_{4}<0$ has the same sign as a "kinetic" term, Equation (20) is like the ellipse equation (Figure 6).

$$
x^{4} / A^{2}+W^{2} / B^{2}=1 .
$$

This is a closed curve around a fixed point $\left(\phi_{s}, 0\right)$, which qualifies it as a stable fixed point.

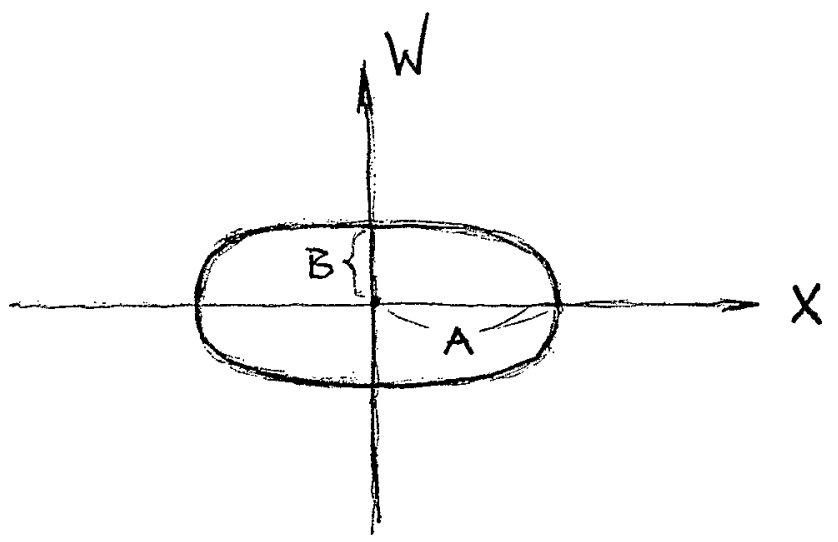

Figure 6. Closed curve (21) around fixed point.

As it was mentioned in Section 2.5, the point $\pi-\phi_{\mathrm{s}}$ is not a fixed pint for a double rf system. We will proceed now to find an unstable fixed point for a double rf system.

\subsection{Unstable Fixed Point}

The unstable fixed point $\phi_{\mathrm{u}}$ possesses two properties. First of all it satisfies $d u / d \phi=0$ or $v\left(\phi_{\mathrm{u}}\right)=v_{\mathrm{s}}$ or

$$
\operatorname{Sin} \phi_{u}+r \operatorname{Sin} 2\left(\phi_{u}-\delta\right)-v_{s}=0,
$$


where $r=4\left(\phi_{\mathrm{s}}\right)$ and $\delta=\delta\left(\phi_{\mathrm{s}}\right)$ satisfy

$$
\begin{aligned}
& \operatorname{Sin} \phi_{s}+r \operatorname{Sin} 2\left(\phi_{s} \delta\right)-v_{s}+0, \\
& \operatorname{Cos} \phi_{s}+2 r \operatorname{Cos} 2\left(\phi_{s}-\delta\right)=0, \\
& \operatorname{Sin} \phi_{s}+4 r \operatorname{Sin} 2\left(\phi_{s}-\delta\right)=0 .
\end{aligned}
$$

Secondly, any trajectory should be open in the vicinity of an unstable fixed point.

Now we will find the unstable fixed point $\phi_{\mathrm{u}}$ using all the properties of the stable fixed point $\phi_{\mathrm{s}}$. Let us introduce the notations:

$$
\left\{\begin{aligned}
\phi_{u} & =\phi_{s}+x, & & y=\operatorname{Sin} x \\
s & =\operatorname{Sin} \phi_{s}, & & S=\operatorname{Sin} 2\left(\phi_{s}-\delta\right) \\
c & =\operatorname{Cos} \phi_{s}, & & C=\operatorname{Cos} 2\left(\phi_{s}-\delta\right)
\end{aligned}\right.
$$

It follows from (23)-(25) that

$$
\begin{aligned}
v_{s} & =\frac{3}{4} s, \\
s c=8 r^{2} S C, \quad r S & =-s / 4, \quad r C=-c / 2, \\
16 r^{2} & =4-3 s^{2} .
\end{aligned}
$$

With use of Eq. (26), we can rewrite the equation for $\phi_{u}$ as

$$
(s-c y) \sqrt{1-y^{2}}=-\frac{s}{2} y^{2}-c y+s .
$$

Here $\sqrt{1-y^{2}}$ represents $\operatorname{Cos} \phi$ and can be positive or negative. As we are going to square (30), it does not mater what sign belongs to $\sqrt{1-y^{2}}$. After squaring both sides of (30) and simplifying, we get:

$$
\left(\frac{s^{2}}{4}+c^{2}\right) y^{2}=s c y^{3},
$$


from where we come to $y=0$ gives trivial solution $\left.\phi_{u}=\phi_{\mathrm{s}}\right)$

$$
y=\frac{4 s c}{s^{2}+4 c^{2}}=\frac{4 \cdot 8 r^{2} S C}{16 r^{2}}=2 S C=\operatorname{Sin} 4\left(\phi_{s}-\delta\right),
$$

or

$$
\operatorname{Sin} x=\operatorname{Sin} 4\left(\phi_{s}-\delta\right) .
$$

This equation gives two values for $\phi_{\mathrm{u}}$ :

$$
\phi_{u}=\phi_{s}+4\left(\phi_{s}-\delta\right)
$$

and

$$
\phi_{u}=\pi+\phi_{s}-4\left(\phi_{s}-\delta\right) .
$$

Let us show that the first one, (34), is not a root of (22). We will need formulae following from (28)

$$
S_{4}=\operatorname{Sin} 4\left(\phi_{s}-\delta\right)=s c / 4 r^{2}, \quad C_{4}=\operatorname{Cos} 4\left(\phi_{s}-\delta\right)=1-s^{2} / 8 r^{2} .
$$

After the substitution of (34) into (22), we get

$$
\begin{gathered}
s C_{4}+c S_{4}+r \operatorname{Sin} 10\left(\phi_{s}-\delta\right)=s C_{4}+c S_{4}+r\left[S\left(C_{4}^{2}-S_{4}^{2}\right)+2 C S_{4} C_{4}\right]= \\
=\frac{-s\left(s^{2}+4\right)\left(13 s^{2}-12\right)}{4\left(4-3 s^{2}\right)^{2}}
\end{gathered}
$$

which is not the expression on the right-hand side of (22).

On the other hand, if (35) is substituted into (22), then one gets

$$
\begin{aligned}
& -s C_{4}+c S_{4}-r \operatorname{Sin} 6\left(\phi_{s}-\delta\right)=-C_{4}(s+r S)+S_{4}(c-r C)= \\
& =-\frac{3 s}{r}\left[1-\frac{s^{2}}{8 r^{2}}\right]+\frac{3 s c^{2}}{8 r^{2}}=\frac{3}{4} s \frac{4 c^{2}+s^{2}-8 r^{2}}{8 r^{2}}=\frac{3}{4} s \frac{16 r^{2}-8 r^{2}}{8 r^{2}},
\end{aligned}
$$

which is exactly equal to the right-hand side of Eq. (22). 
Finally, we have to prove that a fixed point (35) is unstable. We take

$$
\phi=\phi_{u}+x, \quad \phi_{u}=\pi+\phi_{s}-4\left(\phi_{s}-\delta\right)
$$

and having

$$
u(\phi)=\operatorname{Cos} \phi+\frac{r}{2} \operatorname{Cos} 2(\phi-\delta)+\phi v_{s}
$$

we find

$$
\begin{gathered}
u_{1}=\frac{d u\left(\phi_{u}\right)}{d \phi}=0 \\
u_{2}=\frac{d^{2} u\left(\phi_{u}\right)}{d \phi^{2}}=-\operatorname{Cos} \phi_{u}-2 r \operatorname{Cos} 2\left(\phi_{u}-\delta\right)= \\
=c C_{4}+s S_{4}+2 r \operatorname{Cos} 6\left(\phi_{s}-\delta\right)=c C_{4}+s S_{4}+2 r\left(C C_{4}-S S_{4}\right)= \\
=C_{4}(c+2 r C)+S_{4}(s-2 r S)=\frac{3}{2} s S_{4}=\frac{3 s^{2} c}{8 r^{2}}>0 .
\end{gathered}
$$

After substituting (39)-(42) into (10), the later becomes

$$
u_{2} \Delta \phi^{2} / 2 !=-\frac{\pi m}{q V_{o}} \cdot W^{2}+u_{2} x^{2} / 2 !
$$

In this case $u_{2}>0$ has a sign opposite to the sign of the "kinetic" term, which makes (43) a hyperbolic equation

$$
x^{2} / A^{2}-W^{2} / B^{2}=1 .
$$

This is an open trajectory in the nearest vicinity of the fixed point $\left(\phi_{u}, 0\right)$, and for that reason, the fixed point is unstable. 


\section{COMPARISON OF SINGLE AND DOUBLE RF SYSTEMS}

\subsection{Choosing the Basis for Comparison}

In order to compare single and double if systems, we have to choose the basis for comparison. First, we note that the synchronous voltage from (2.8) and (2.11) is

$$
V_{s}=V_{o} \cdot v\left(\phi_{s}\right)=2 \pi R \rho \dot{B}
$$

which determines the rate of acceleration. This voltage should be the same for both systems. This means that both systems are referring to the same moment of the accelerating cycle. At the same time, peak voltages can be different for the single $V_{o}=V_{01}$ and for the double $V_{o}=V_{02}$ rf system. The fact of equality of the two synchronous voltages can be expressed by

$$
V_{01} v_{s 1}=V_{02} v_{s 2}=V_{s} \text {. }
$$

Second, we note that there are many other parameters that can be equalized for the single and double system. For instance, two important parameters of bucket and bunch are its height (or half-height) and area. Height and area both depend on the peak voltage. Both of them have advantages and disadvantages if used under the limitations imposed by various accelerator conditions such as available aperture, maximal magnetic field or field rate, stability thresholds, and so on.

Because the physical aperture of the vacuum chamber is the most severe limitation on the bunch/bucket parameters, and because bunch (and bucket) height is directly restricted by the aperture and because the mathematics of height is much easier than that of area, we choose the bucket height $h_{b}$ as a common basis for comparison of the single and double rf systems:

$$
h_{b 1}=h_{b 2} \text {. }
$$

As we will see later in section 4.3, our two conditions (1) and (2) uniquely determine interdependence of two peak voltages-- $V_{01}$ for the single system and $V_{02}$ for the double system. This means that if, lets say, $V_{01}$ is given then in order to make (1) and (2) valid, the second voltage $V_{02}$ should be a certain function of the first one:

$$
V_{02}=P\left(V_{01}\right)
$$


We will now proceed from the several definitions of bucket parameters to derive Equation (3), after which we will be equipped for the logically founded comparison of the two systems.

\subsection{The Bucket and Its Height}

As we have seen in a phase plane $(\phi, W)$ all the fixed points align along the $\phi$-axis, whose equation is $W=0$. Every stable trajectory crosses the $\phi$-axis at least once. Any point, $\psi$, of the $\phi$-axis corresponds to a certain trajectory. There could be more than one point, $\psi_{1}, \psi_{2}, \ldots$ belonging to one trajectory. However, it is enough to peak up one point $\psi$ in order to select a single trajectory, because every single point $\psi$ determines the value of the Hamiltonian (3.1) for the particle crossing that point $\phi=\psi, W=0$ :

$$
H(\psi, 0)=-\frac{q}{2 \pi} U(\psi),
$$

which in turn determines the trajectory equation on the phase plane $(\phi, W)$ :

$$
-\frac{q}{2 \pi} U(\psi)=-m \frac{W^{2}}{2}-\frac{q}{2 \pi} U(\phi),
$$

or due to $(2.14)$

$$
W(\phi, \psi)= \pm \sqrt{\frac{q}{\pi m}[U(\psi)-U(\phi)]}= \pm \sqrt{\frac{q V_{o}}{\pi m}[u(\phi)-u(\psi)]}
$$

We call parameter $\psi$ a phase amplitude of the particle's trajectory or just amplitude. We call parameter

$$
h_{s}=\sqrt{\frac{q V_{o}}{\pi m}}
$$

a synchronous half-height because it depends on the synchronous energy $E_{s}$. If $\phi_{\mathrm{s}}$ is a stable fixed point, then we call parameter

$$
h_{\psi}=\sqrt{u\left(\phi_{s}\right)-u(\psi)}
$$

a normalized half-height of the particle's trajectory of amplitude $\psi$ or simply the normalized half-height. Finally, we call

$$
w(\phi, \psi)= \pm \sqrt{u(\phi)-u(\psi)}
$$


a normalized particle's trajectory (of amplitude $\psi$ ), so (2) becomes

$$
W(\phi, \psi)=h_{s} w(\phi, \psi)= \pm h_{s} \cdot \sqrt{u(\phi)-u(\psi)} .
$$

If the trajectory crosses the unstable fixed point $\left(\psi=\phi_{\mathrm{u}}\right)$, then (6) becomes an equation of separatrix

$$
S(\phi)=W\left(\phi, \phi_{u}\right)= \pm h_{s} \cdot \sqrt{u(\phi)-u_{u}}, \quad u_{u}=u\left(\phi_{u}\right) .
$$

We call a normalized separatrix the curve

$$
s(\phi)=w\left(\phi, \phi_{u}\right)= \pm \sqrt{u(\phi)-u_{u}} .
$$

A closed part of the (normalized) separatrix encompasses the phase plane area called the (normalized) bucket. The half-height of the normalized bucket is

$$
h_{n}=\sqrt{u_{s}-u_{u}} \text {. }
$$

The unnormalized bucket half-height is

$$
h_{b l t}=h_{s} \cdot h_{n} \text {. }
$$

For the single rf system

$$
\left\{\begin{aligned}
u(\phi) & =\operatorname{Cos} \phi+\phi v_{s}, \\
u_{s}-u_{u} & =2 \operatorname{Cos} \phi_{s}-\left(\pi-2 \phi_{s}\right) v_{s} .
\end{aligned}\right.
$$

For the double rf system

$$
u_{s}-u_{u}=\operatorname{Cos} \phi_{s}\left(2+\frac{1}{16 r^{2}}\right)-\left[\pi-4\left(\phi_{s}-\delta\right)\right] v_{s} .
$$

\subsection{Voltage Connection for the Two Systems and Data for the Power Supply}

We will now derive the equation connecting the synchronous phases of the single and double rf systems each of which creates a bucket with the same height. In all of the following formulae, the index 1 refers to the single system and the index 2 is for the double system, so our goal is to find a function connecting the two phases $\phi_{o 2}=F\left(\phi_{o 1}\right)$. 
Because both systems have the same synchronous voltage (accelerating rate) $V_{\mathrm{s}}$, we can write

$$
V_{o 1} v_{s 1}=V_{o 2} v_{s 2}
$$

where the wave voltages at the synchronous angle according to (2.21) are

$$
\left\{\begin{array}{l}
v_{s 1}=v_{1}\left(\phi_{s 1}\right)=\operatorname{Sin} \phi_{s 1}=s_{1}, \\
v_{s 2}=v_{2}\left(\phi_{s 2}\right)=\frac{3}{4} \cdot \operatorname{Sin} \phi_{s 2}=\frac{3}{4} s_{2} .
\end{array}\right.
$$

Because both systems create buckets of the same height, we have from (6), (13)-(15)

$$
V_{o 1}\left(u_{s 1}-u_{u 1}\right)=V_{o 2}\left(u_{s 2}-u_{u 2}\right)
$$

or due to $(2.15),(15)$

$$
V_{o 1}\left(2 \operatorname{Cos} \phi_{s 1}+2 \phi_{s 1} V_{s 1}\right)=V_{o 2} \operatorname{Cos}_{s 2}\left(2+\frac{1}{16 r^{2}}\right]+4\left(\phi_{s 2}-\delta\right) V_{s}
$$

or with the use of (1) and (2.32)

$$
\operatorname{ctg} \phi_{s 1}+\phi_{s 1}=\frac{2}{3} \operatorname{ctg} \phi_{s 2} \cdot \frac{8+3 \operatorname{tg}^{2} \phi_{s 2}}{4+3 \operatorname{tg}^{2} \phi_{s 2}}+\operatorname{arctg} \frac{\operatorname{tg} \phi_{s 2}}{2} .
$$

This equation of the type $F\left(\phi_{s 1}, \phi_{s 2}\right)=0$ implicitly connects synchronous phases for the two systems of equal bucket height.

With the use of any numerical method, the implicit equation can be solved and the function $\phi_{o 2}=F\left(\phi_{o 1}\right)$ can be constructed. After that the voltage connection $V_{o 2}=P\left(V_{o 1}\right)$ can be recovered also numerically (or with a monstroustype formulae analytically). Figure 7 shows a graph of the function $\phi_{o 2}=F\left(\phi_{01}\right)$. With the use of this function, we have constructed a function $v_{s 2}=P\left(v_{s 1}\right)$ shown in Figure 8. As peak voltage for each system can be expressed as

$$
V_{o i}=\frac{V_{s}}{v_{s i}}, \quad i=1,2 \text {, }
$$


a function $V_{o 2}=P\left(V_{o 1}\right)$ can be recovered from a function $P$. Instead, we have constructed a function $a=a\left(v_{s 1}\right)$ :

$$
a=\frac{V_{01}-V_{o 2}}{V_{o 1}},
$$

which does not require a use of accelerating rate $V_{\mathrm{s}}$. A function $a$ is shown in Figure 9 and Table I. The meaning of this function is that if you know the peak voltage $V_{01}$ for the single rf system, then you have for the double system a relative deviation $a$ of peak voltage

$$
V_{o 2}=(1-a) V_{o 1},
$$

which would provide a bucket with the same height as for the single system. As $a$ is always non positive $(a \leq 0)$, then

$$
V_{o 2} \geq V_{o 1} \text {. }
$$

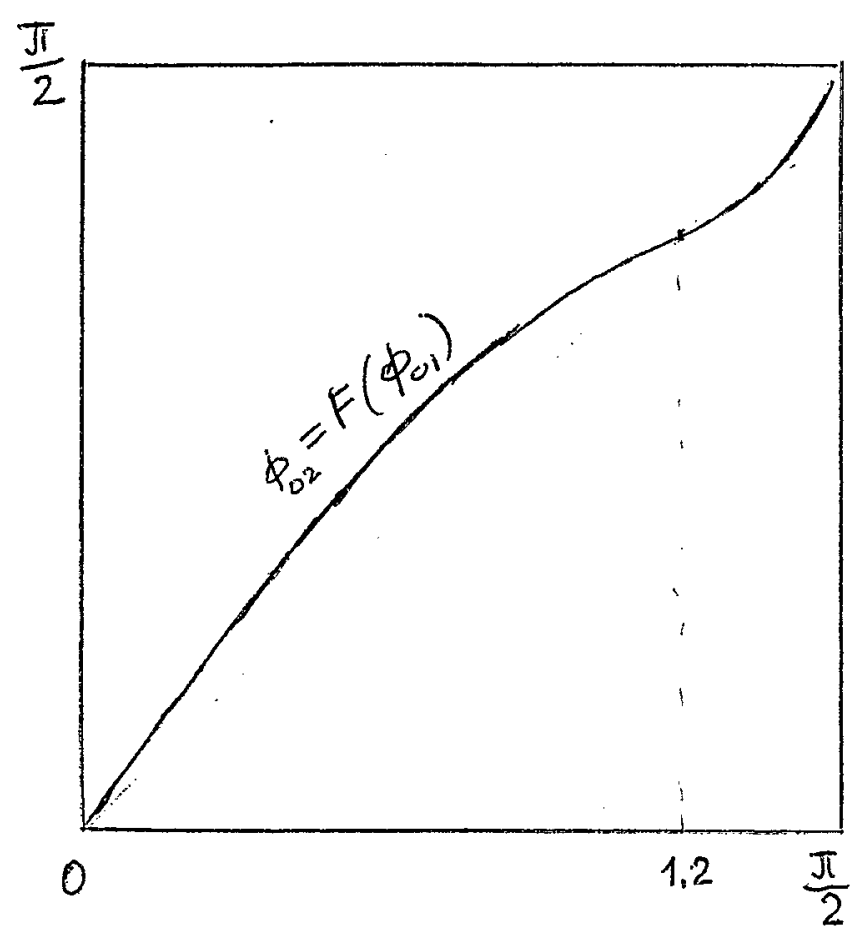

Figure 7

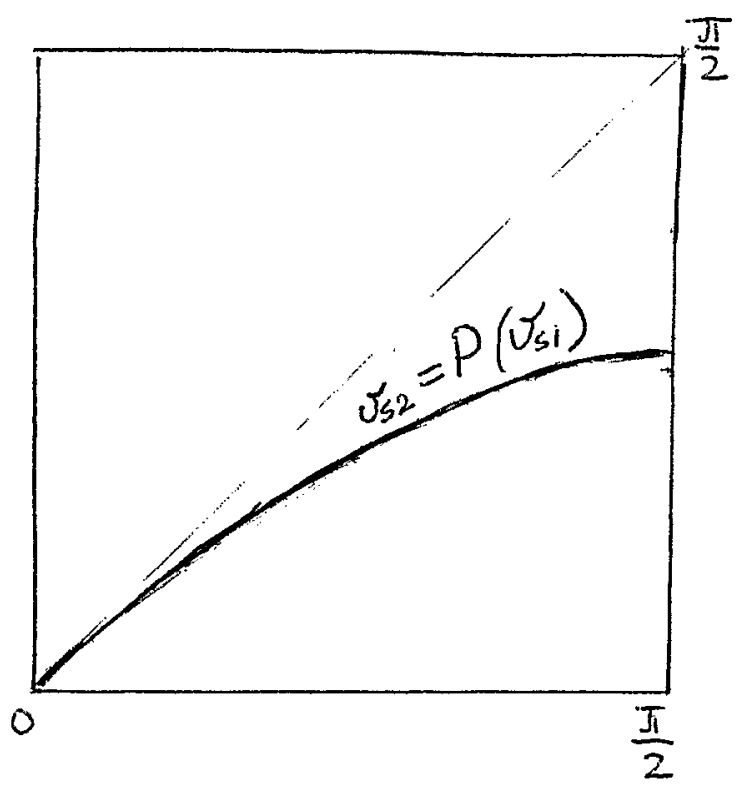

Figure 8 


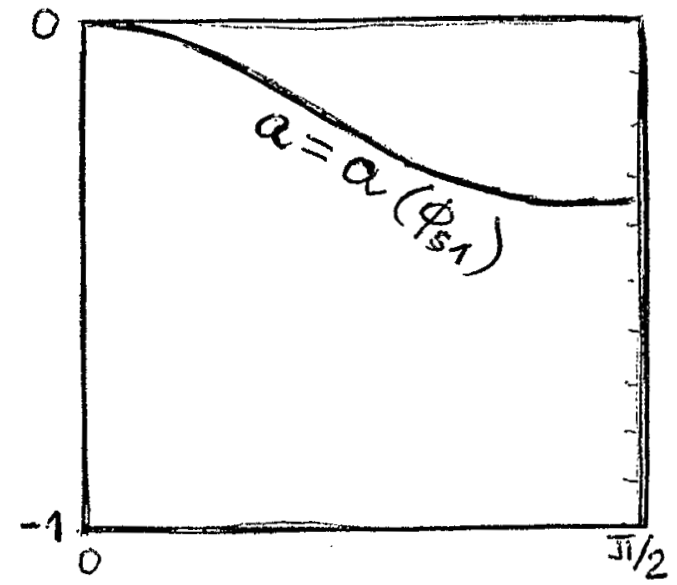

Figure 9

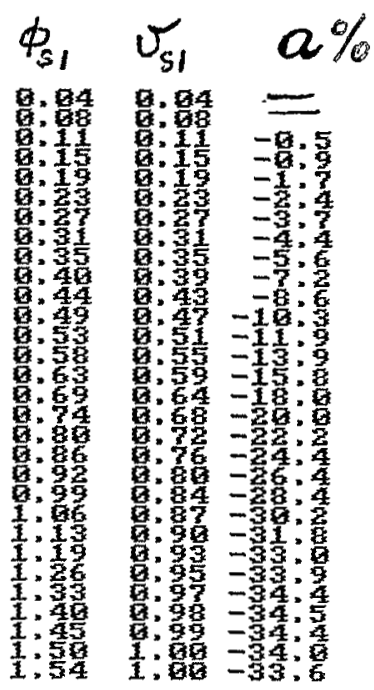

Table I

The peak voltage is an important parameter not only for particle dynamics, but also for designing if cavities and for the cost evaluation of the whole accelerating cycle. One aspect of the accelerator's total power consumption is the amount of rf applied voltage $V_{a}$. The applied voltage is the same as the peak voltage for the single rf system $V_{a 1}=V_{o 1}$. For the double if system, the applied voltage is the sum of the peak voltages applied for the first and for the second harmonic cavities, while the total voltage acting upon the particle is a combination (2.5a), (2.5b):

$$
V_{o 2}[\operatorname{Sin} \phi+r \operatorname{Sin} 2(\phi-\delta)],
$$

where $V_{02}$ is the peak voltage for the first harmonic, while $|r| V_{o 2}$ is the peak voltage for the second harmonic. Thus, the applied voltage is

$$
V_{a 2}=(1+|r|) V_{o 2} \text {. }
$$

After the substitution of (22) into (25), we have

$$
V_{a 2}=(1+|r|)(1-a) V_{a 1} \text {. }
$$

This result can be used as data for the evaluation of power supplies for the double rf system. 


\subsection{Bucket Length and Area}

Bucket length and area are important parameters when we are trying to evaluate injection and capture efficiency. The bucket length is a distance in phase plane $(\phi, W)$ between two points where the separatrix intersects the $\phi$-axis (Figure 10). One point is an unstable fixed point $\phi=\phi_{u}$, another one is the so-called separatrix extreme point $\phi=\phi_{e}$, which is a root of the equation

$$
u\left(\phi_{e}\right)=u\left(\phi_{u}\right) .
$$

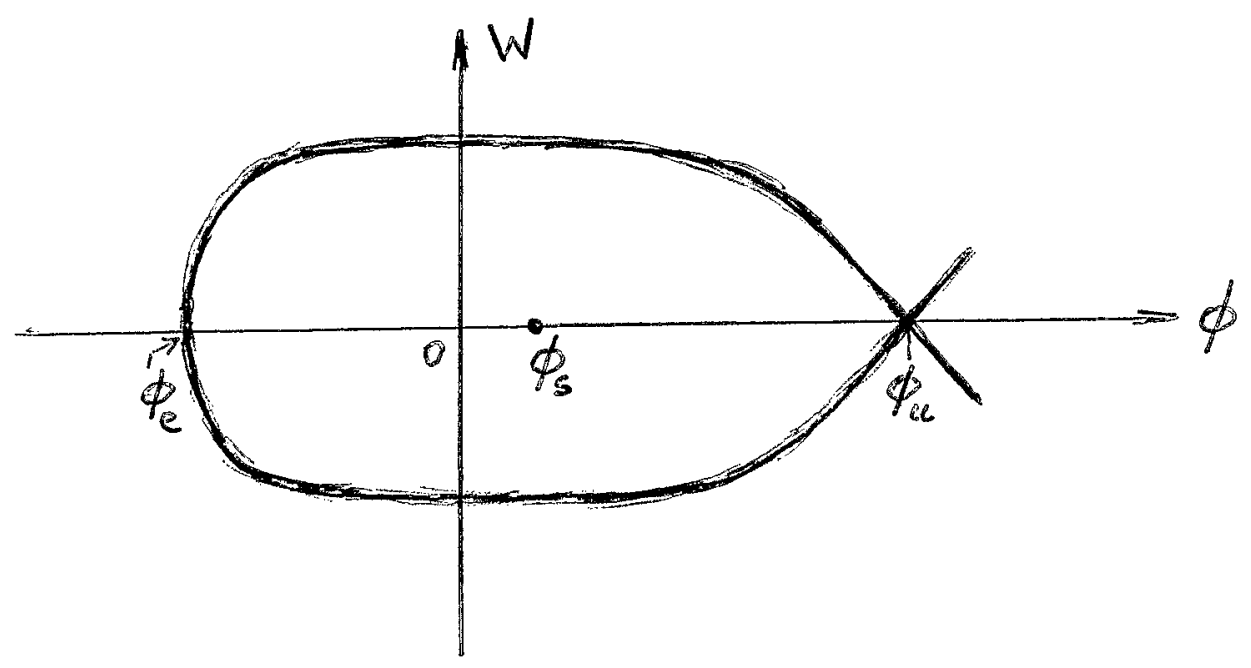

Figure 10.

For the single rf system

$$
u(\phi)=\operatorname{Cos} \phi+\phi v_{s}, \quad \phi_{u}=\pi-\phi_{s}, \quad v_{s}=\operatorname{Sin} \phi_{s} .
$$

For the double rf system (2.15)

$$
u(\phi)=\operatorname{Cos} \phi+\frac{r}{2} \operatorname{Cos} 2(\phi-\delta)+\phi v_{s}, \phi_{u}=\pi+\phi_{s}-4\left(\phi_{s}-\delta\right), v_{s}=\frac{3}{4} \operatorname{Sin} \phi_{s},
$$

where $r$ and $\delta$ are defined according to (2.24) and (2.25). The bucket length can now be expressed as

$$
\ell=\phi_{u}-\phi_{e}
$$


The bucket area is the phase plane area within the closed part of the separatrix:

$$
A=2 \int_{\phi_{e}}^{\phi_{u}} S(\phi) d \phi=2 h_{s} \int_{\phi_{e}}^{\phi_{u}} \sqrt{u(\phi)-u\left(\phi_{u}\right)} d \phi=2 h_{s} \alpha_{n},
$$

where $h_{s}$ is the bucket's synchronous half-height (6) while $\alpha_{n}$ is the bucket's normalized area.

Equation (27) can be solved and integral (31) can be evaluated numerically for any set of input parameters. After that we can compare lengths and areas of buckets with equal heights for the single and double rf systems at any accelerating rate.

Figure 11 shows graphs of $\phi_{e}, \phi_{u}$ and $\ell$ for the single and double systems as a function of the synchronous phase $\phi_{\mathrm{s} 1}$. Figure 12 shows the relative deviations

$$
\delta \ell=\frac{\ell_{2}-\ell_{1}}{\ell_{1}} \cdot 100 \%, \quad \delta A=\frac{A_{2}-A_{1}}{A_{1}} \cdot 100 \%
$$

in bucket length and area for the double system with respect to the single system.

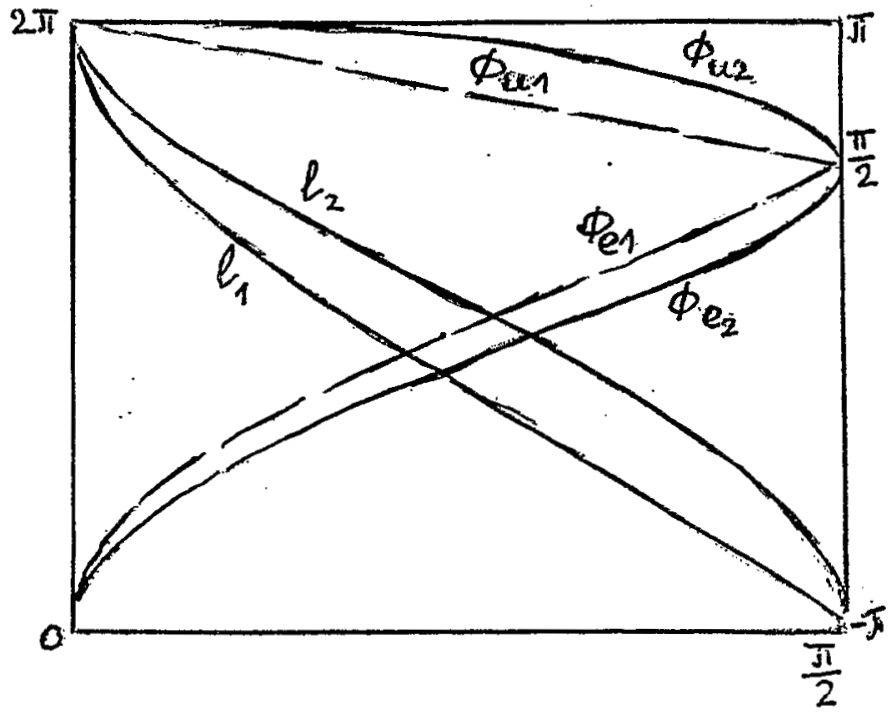

Figure 11.

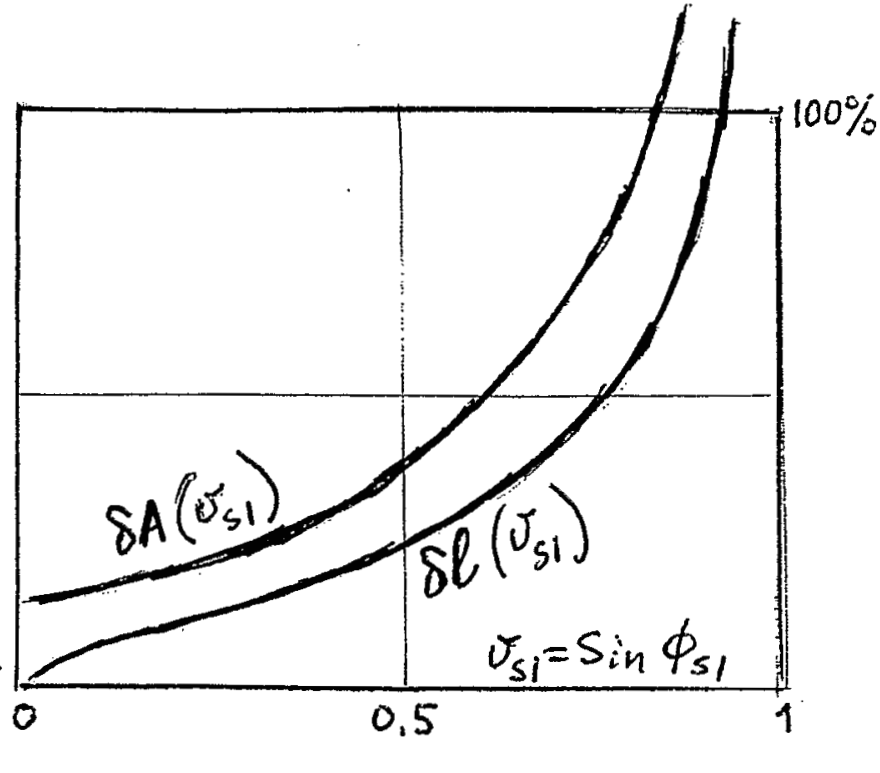

Figure 12. 


\subsection{Synchrotron Frequency}

Let us consider an arbitrary particle within the bucket that possesses only one stable fixed point. All trajectories within the bucket are closed. Due to closeness,particle motion will be periodic with time. The frequency corresponding to this motion is called synchrotron frequency.

Let $\psi$ be a particle's intersection as it is defined in Section 4.2. this means that the particle's trajectory intersects the $\phi$-axis at the point $\phi=\psi$ (Figure 13). Because the trajectory is closed, it will intersect the $\phi$-axis at a second point too. To distinguish the two intersections, we denote the right one as $\psi_{\mathrm{r}}$ and the left one as $\psi_{\ell}$.

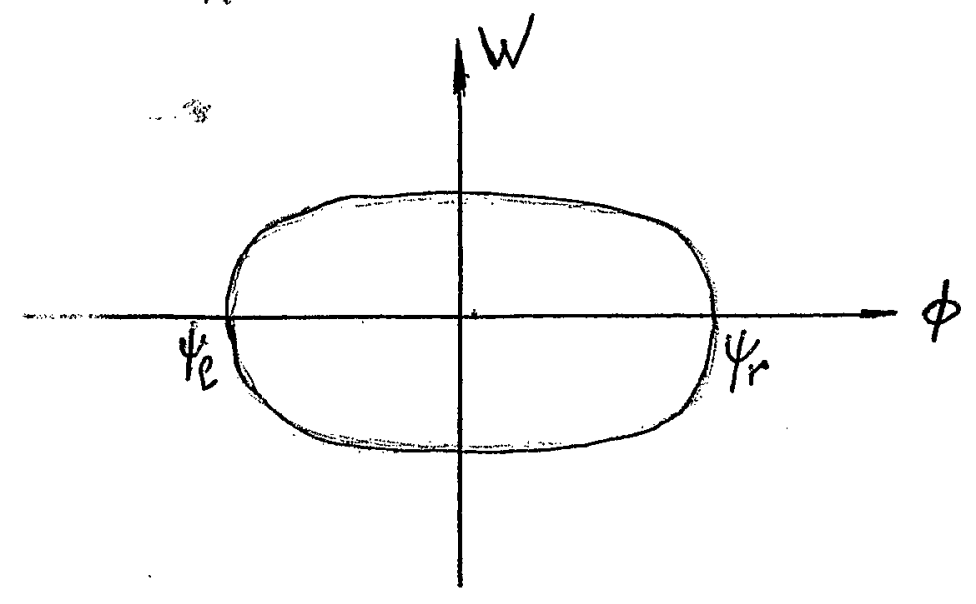

Figure 13. Particle's trajectory with two intersections.

According to (4.9), the particle's trajectory can be expressed as

$$
\begin{aligned}
W\left(\phi, \psi_{r}\right) & = \pm h_{s} \cdot \sqrt{u(\phi)-u\left(\psi_{r}\right)}= \\
& = \pm h_{s} \cdot \sqrt{u(\phi)-u\left(\psi_{\ell}\right)}=W\left(\phi, \psi_{\ell}\right) .
\end{aligned}
$$

If one of the two intersections $\psi_{\ell}, \psi_{\mathrm{r}}$ is known, then another one can be found from

$$
u_{\ell} \equiv u\left(\psi_{\ell}\right)=u\left(\psi_{r}\right) \equiv u_{r}
$$


Suppose we know $\psi_{\mathrm{r}}, \psi_{\ell}$ for some particle. By taking Equation (2.1)

$$
\frac{d \phi}{d t}=-m W
$$

and rewriting it as

$$
\frac{d t}{d \phi}=\frac{1}{-m W}
$$

we can express the period $T$ of synchrotron oscillations for the particle in question. It follows from (35) that when $W>0$, then the particle moves from right to left, and vice versa (because $m>0$ ). For this reason, we integrate (36) from the right intersection to the left one, and for the full period we double the integral:

$$
T=2 \int_{\psi_{r}}^{\psi_{t}} \frac{d \phi}{-m W\left(\phi, \psi_{r}\right)}
$$

or by reversing the path of integration, we get

$$
T=\frac{2}{m h_{s}} \cdot \int_{\psi_{\ell}}^{\psi_{r}} \frac{d \phi}{\sqrt{u(\phi)-u_{r}}},
$$

where $m$ is defined in (2.3), and the synchronous bucket half-height $h_{\mathrm{s}}$ is defined according to (6). After the period $T$ is found, a synchrotron frequency $F$ can be written:

$$
F=\frac{1}{T}
$$

Formula (38) is equally applicable to the single and double rf systems. 


\subsubsection{Synchrotron Frequency of Small Amplitude}

The distances between trajectory intersections and the stable fixed point

$$
\Delta \psi_{r}=\psi_{r}-\phi_{s}, \quad \Delta \psi_{\ell}=\phi_{s}-\psi_{\ell}
$$

are called right and left amplitudes. They are equal if the bucket is stationary: $\phi_{\mathrm{s}}=0$. They are approximately equal when

$$
\psi_{r}-\psi_{\ell} \ll 1 \text {. }
$$

Then the right and left amplitudes are

$$
\Delta \psi_{r}=\Delta \psi_{\ell}=\Delta \phi
$$

and we call them just small amplitude.

The case of small amplitude synchrotron frequency can be expressed by a simple analytical formula. We will derive it now for the single and double rf systems.

Single RF System

For this case, the potential wave is

$$
u(\phi)=\operatorname{Cos} \phi+\phi v_{s},
$$

where $v_{s}=\operatorname{Sin} \phi_{s}$. Let us introduce a new variable by putting

$$
\phi=\phi_{s}+x
$$

After Taylor expansion of the potential wave $u(\phi)$, we have

$$
u\left(\phi_{s}+x\right)=u_{s}+u_{1} x+u_{2} x^{2} / 2 !+\ldots,
$$

where $u_{s}=u\left(\phi_{s}\right), \quad u_{i}=d^{i} u\left(\phi_{s}\right) / d \phi^{i}, \quad i=1,2, \ldots$. 
According to (3.5) the fixed point $\phi_{\mathrm{s}}$ satisfies $u_{1}=0$, which leaves us

$$
u(\phi)=u\left(\phi_{s}+x\right)=u_{s}+u_{2} x^{2} / 2,
$$

where $u_{2}=-\operatorname{Cos} \phi_{s}$. When $x=\Delta \phi=\phi_{r}-\phi_{s}$, then

$$
u_{r}=u\left(\phi_{s}+\Delta \phi\right)=u_{s}-\Delta \phi^{2} \operatorname{Cos} \phi_{s} / 2 .
$$

As (45) can be split into two sign-alternating series and

$$
\begin{aligned}
& u_{3}=-\operatorname{Sin} \phi_{s}, \quad u_{4}=\operatorname{Cos} \phi_{s}, \text { then } \\
& \Delta u=\left|u(\phi)-\left(u_{s}+u_{1} x+u_{2} x^{2} / 2 !\right)\right| \leq \Delta \phi^{3} \operatorname{Sin} \phi_{s} / 6+\Delta \phi^{4} \operatorname{Cos} \phi_{s} / 24 .
\end{aligned}
$$

Thus, after replacement $u(\phi)$ by its Taylor expansion up to the second order, the absolute error $\Delta u$ will be less than $\epsilon$ if the inequality satisfies

$$
\Delta \phi^{3} \operatorname{Sin} \phi_{s} / 6+\Delta \phi^{4} \operatorname{Cos} \phi_{s} / 24 \leq \epsilon,
$$

or

$$
\Delta \phi \leq \sqrt[3]{\frac{6 \epsilon}{\operatorname{Sin} \phi_{s}+\frac{1}{4} \operatorname{Cos} \phi_{s}}}
$$

This is the criteria of smallness of the phase amplitude $\Delta \phi$.

After substituting (46) and (47) into (38), the period $T$ will be written as

$$
T=\frac{4}{m h_{s}} \cdot \int_{0}^{\Delta \phi} \frac{d x}{\sqrt{0.5 \Delta \phi^{2} \operatorname{Cos} \phi_{s}-0.5 x^{2} \operatorname{Cos} \phi_{s}}}=\frac{2 \pi}{m h_{s}} \sqrt{\frac{2}{\operatorname{Cos} \phi_{s}}},
$$

and taking $m$ from (2.1), and $h_{s}$ from (6), we have the synchrotron frequency

$$
F=\frac{1}{T}=\frac{\omega_{s}}{2 \pi} \sqrt{\frac{q V_{o} h \eta \operatorname{Cos} \phi_{s}}{2 \pi \beta E_{s}^{2}}}=f_{s} v
$$


where

$$
f_{s}=\frac{\omega_{s}}{2 \pi}
$$

is the particle's revolution frequency and

$$
v=\sqrt{\frac{q V_{o} h \eta \operatorname{Cos} \phi_{s}}{2 \pi \beta^{2} E_{s}}} .
$$

is the synchrotron tune.

Double RF System

For this case, the potential wave is

$$
u(\phi)=\operatorname{Cos} \phi+\frac{r}{2} \operatorname{Cos} 2(\phi-\delta)+\phi v_{s}, \quad v_{s}=\frac{3}{4} \operatorname{Sin} \phi_{s} .
$$

and its Taylor expansion is $\left(u_{i}=d^{i} u\left(\phi_{s}\right) / d \phi^{i}\right)$ :

$$
u(\phi)=u\left(\phi_{s}+x\right)=u_{s}+u_{1} x+u_{2} x^{2} / 2 !+u_{3} x^{3} / 3 !+u_{4} x^{4} / 4 !+\ldots
$$

Due to (3.17) and (3.18), $u_{1}=u_{2}=u_{3}=0$ and $u_{4}=-3 \operatorname{Cos} \phi_{s}$, which gives

$$
u(\phi)=u_{s}-x^{4} \operatorname{Cos} \phi_{s} / 8
$$

and

$$
u\left(\phi_{r}\right)=u\left(\phi_{s}+\Delta \phi\right)=u_{s}-\Delta \phi^{4} \operatorname{Cos} \phi_{s} / 8
$$

The series (56) can be split into two sign-alternating series and $u_{5}=-3 \operatorname{Sin} \phi_{s}, u_{6}=-15 \operatorname{Cos} \phi_{s}$, then

$$
\begin{aligned}
\Delta u=\left|u(\phi)-\left(u_{s}+u_{4} x^{4} / 4 !\right)\right| & \leq \Delta \phi^{5} \operatorname{Sin} \phi_{s} \cdot \frac{3}{5 !}+\Delta \phi^{6} \operatorname{Cos} \phi_{s} \cdot \frac{15}{6 !} \leq \\
& \leq 2 \Delta \phi^{5} \operatorname{Sin} \phi_{s} \cdot \frac{3}{5 !} .
\end{aligned}
$$


Thus, after replacement of $u(\phi)$ by its Taylor expansion up to the fourth order, the absolute error $\Delta u$ will be less than $\epsilon$ if the inequality satisfies

$$
\Delta \phi^{5} \operatorname{Sin} \phi_{s} / 20+\Delta \phi^{6} \operatorname{Cos} \phi_{s} / 48 \leq \epsilon,
$$

or

$$
\Delta \phi \leq \sqrt[5]{\frac{240 \epsilon}{6 \operatorname{Sin} \phi_{s}+5 \operatorname{Cos} \phi_{s}}} .
$$

This is the criteria of smallness of the phase amplitude $\Delta \phi$.

Let $\Delta \phi$ be a small bunch half-length. By taking from (50) and (61) only the equalities, we can consider a function

$$
\Delta \phi=\Delta \phi(\epsilon)
$$

for a single rf as well as for a double rf when both are adjusted for buckets of equal height. Figure 14 shows a comparison of two half-lengths $\Delta \phi$ for small bunches at various value of error $\epsilon$.

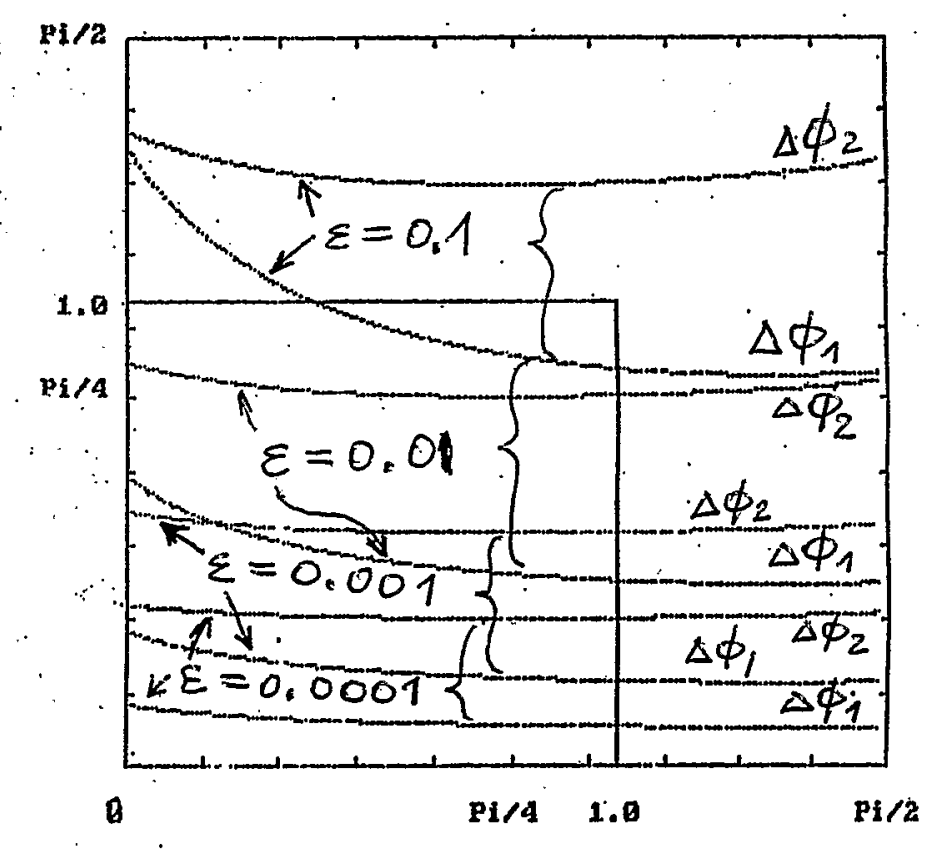

Figure 14. Comparison of two bunch lengths. 
After substituting (57) and (58) into (38), period $T$ will be written as

$$
\begin{aligned}
T & =\frac{2}{m h_{s}} \cdot \int_{\psi_{t}}^{\psi_{r}} \frac{d \phi}{\sqrt{u(\phi)-u_{r}}}=\frac{8}{m h_{s}} \cdot \sqrt{\frac{2}{\operatorname{Cos} \phi_{s}}} \int_{o}^{\Delta \phi} \frac{d x}{\sqrt{\Delta \phi^{4}-x^{4}}}= \\
& =\frac{8}{m h_{s} \Delta \phi \sqrt{\operatorname{Cos} \phi_{s}}} \cdot F\left[\frac{\pi}{2}, \frac{\sqrt{2}}{2}\right],
\end{aligned}
$$

where $F\left(\frac{\pi}{2}, \frac{\sqrt{2}}{2}\right)=K\left(\frac{\sqrt{2}}{2}\right)=1.854$ is the complete elliptical integral of the

first kind. Now we get for the synchrotron frequency

$$
F=\frac{1}{T}=\frac{\omega_{s} \Delta \phi}{8 \cdot 1.854} \sqrt{\frac{q V_{o} h \eta \operatorname{Cos} \phi_{s}}{\pi \beta^{2} E_{s}}}
$$

where $f_{s}=\frac{\omega_{s}}{2 \pi}$ is the particle's revolution frequency and

$$
v=\frac{\pi \Delta \phi}{4 \cdot 1.854} \sqrt{\frac{q V_{o} h \eta \operatorname{Cos} \phi_{s}}{\pi \beta^{2} E_{s}}}
$$

is the synchrotron tune.

The important difference between the single and double rf systems is that the latter has synchrotron frequency and tune vanishing at the center of the bunch $(\Delta \phi=0)$.

\subsubsection{Synchrotron Frequency of Finite Amplitude}

When amplitudes $\Delta \psi_{r}, \Delta \psi_{\ell}$ are finite, not small, then the synchrotron period, frequency, and tune are evaluated numerically from the integral

$$
T=\frac{2}{m h_{s}} \cdot \int_{\psi_{\ell}}^{\psi_{r}} \frac{d \phi}{\sqrt{u(\phi)-u_{r}}}, \quad F=\frac{1}{T}, \quad v=2 \pi \frac{F}{\omega_{s}} .
$$




\subsection{Bunching Factor}

As we have seen in Section 1, instabilities in particle flow can occur when inhomogeneity

$$
d I / d \phi / I(\phi)=d \lambda / d \phi / \lambda(\phi)
$$

in the current $I$ or in the line density $\lambda$ will become relatively high. This can happen at any point $\phi$ within the bunch $\phi_{\ell} \leq \phi \leq \phi_{r}$. Experimentally it would be extremely inconvenient or impossible to examine criteria (67) at every point $\phi$ with acceptable accuracy. That is why instead of local criteria (67), a concept of the bunching factor as a global criteria was developed allowing the characterization of a whole bunch with respect to its inhomogeneity.

The bunching factor $B$ is defined as the ratio of the average $\bar{\lambda}$ to

peak $\lambda_{\max }$ line density $\lambda=\lambda(\phi), \quad \phi_{\ell} \leq \phi \leq \phi_{r}$ :

$$
B=\bar{\lambda} / \lambda_{\max },
$$

where $\phi_{\ell}$ and $\phi_{r}$ are the left and right boundary of the bunch, and

$$
\begin{gathered}
\bar{\lambda}=\frac{1}{\phi_{r}-\phi_{\ell}} \int_{\phi_{\ell}}^{\phi_{r}} \lambda(\phi) d \phi, \\
\lambda_{\max }=\max _{\phi} \lambda(\phi) .
\end{gathered}
$$

For example, in Figure 15, if $\phi_{r}-\phi_{\ell}=2 \Delta \phi\left(-\phi_{r}=\Delta \phi=\phi_{\ell}\right)$ and

$$
\lambda(\phi)=1-\left|\frac{\phi}{\Delta \phi}\right|^{2 n},
$$

then the bunching factor will change with $n$ as it is shown in Table II. 


\begin{tabular}{|c|c|c|c|c|}
\hline \hline $\mathrm{n}$ & $1 / 2$ & 1 & 2 & 3 \\
\hline $\mathrm{B}$ & $1 / 2$ & $2 / 3$ & $4 / 5$ & $6 / 7$ \\
\hline
\end{tabular}

Table II

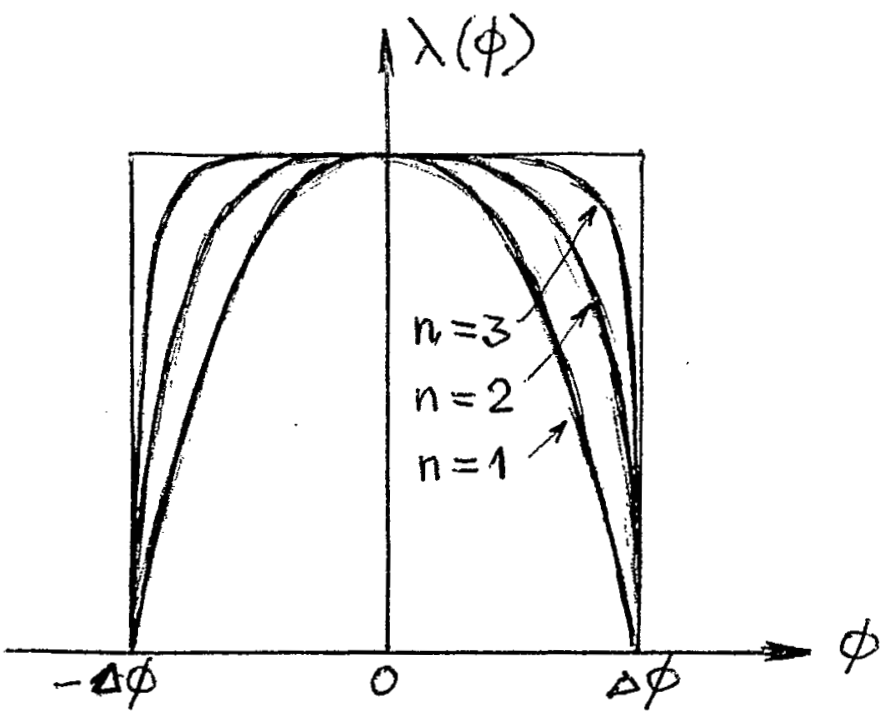

Figure 15. Line density as a parabolic function of various order $n$.

Following Hofmann and Pedersen [9], we will choose the phase density

$$
\rho(H)=\text { const } \cdot \sqrt{H_{b}-H} .
$$

Such a choice being general enough for proton applications, provides at the same time a convenient form for the line density $\lambda$ :

$$
\lambda(\phi)=\text { const } \cdot\left[u(\phi)-u\left(\phi_{l}\right)\right],
$$

where $\phi_{\mathrm{u}}$ is an unstable fixed point, and $\mathrm{u}$ is the potential determined by (28) for the single rf system or by (29) for the double system.

The left point of bunch $\phi_{\ell}=\phi_{e}$ is the extreme point determined according to Equation (27). The right point $\phi_{r}=\phi_{u}$ is an unstable fixed point. From (73) it is obvious that the maximal value of $\lambda$ will be reached at the stable fixed point $\phi_{s}$, where $\lambda(\phi)=u(\phi)=0$. 
Now the bunching factor B for the line density (73) can be written as follows

$$
B=\bar{\lambda} / \lambda_{\max }=\frac{\int_{\phi_{e}}^{\phi_{u}}\left[u(\phi)-u_{u}\right] d \phi}{\left(\phi_{u}-\phi_{e}\right)\left(u_{s}-u_{u}\right)} .
$$

Figure 16 shows $B_{1}$ and $B_{2}$ the bunching factors for the single and double rf systems when the corresponding buckets have the same height. There is also a relative deviation

$$
\delta B=\frac{B_{2}-B_{1}}{B_{1}} \cdot 100 \%
$$

As we can see, the double rf system provides a bunching factor larger than the single system by $23-28 \%$ throughout the whole region.

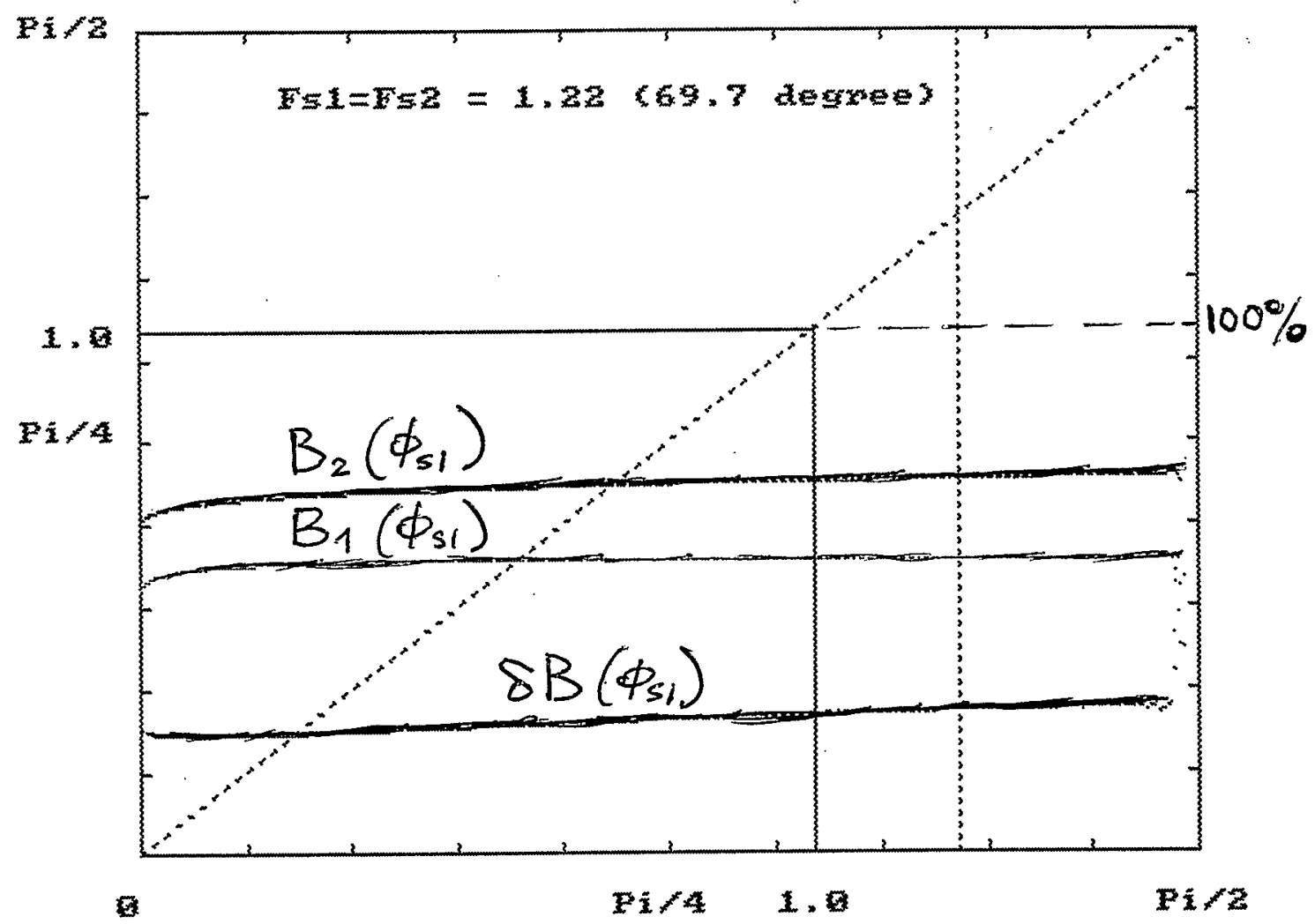

Figure 16. 


\subsection{Capture Efficiency}

Injection from the linac to the circular machine with subsequent capture is a pretty complicated process. The capture efficiency depends on many factors of different natures. Some of them are

- $\quad$ Capture program $V(t), F(t), \dot{B}(t)$-- voltage, frequency, magnetic field;

- Linac program $E(t), \Delta E, \Delta t$-injection energy, energy spread, duration;

- Chopper program $\Delta \phi(t)$ - length and composition of chopped strips;

- Available aperture;

- $\quad$ Bucket parameters.

We will use the last factor here for a rough estimation of the capture efficiency or more precisely for comparison of the capture efficiency between the single and double rf systems with the same bucket height. Let us consider injection and capture for two extreme cases:

(a) Injected beam has a very small energy spread $\Delta \mathrm{E}$,

(b) Injected beam has a very large energy spread $\Delta \mathrm{E}$.

The real case will always be somewhere between (a) and (b). Obviously, in the first case, the capture efficiency will be proportional to the bucket length, while in the second case, the capture efficiency will be proportional to the bucket area. Thus, from Figure 17 we can see a strip between the two curves, one of which is the relative deviation in bucket length. then, the relative deviation in the capture efficiency for the double system compared to the single system will be within the strip.

Figure 17.

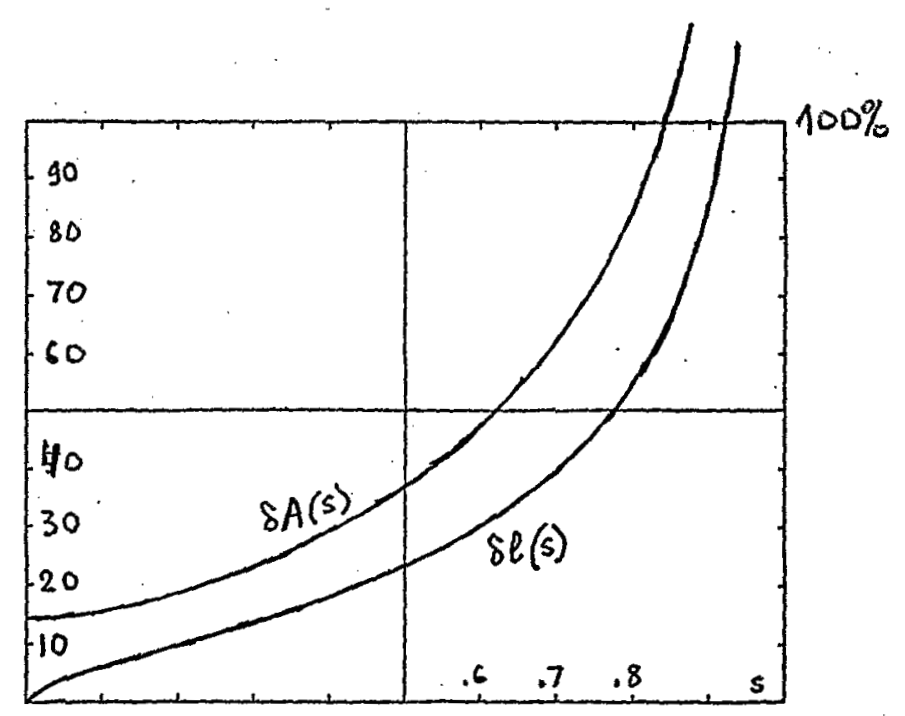




\section{ACKNOWLEDGMENTS}

I am thankful to Dr. W.T. Weng for suggesting to me the investigation

of the double rf system. I am grateful to $M$. Heimerle for her work in preparing the manuscript.

\section{REFERENCES}

[1] P.I. Lebedev, L.Z. Barabash, A.V. Barkhudarjan, L.L. Goldin, Yu.M. Zlatov, "Use of Nonsinusoidal Shaped Accelerating Voltage to Increase Particle Capture Efficiency in ITEP Proton Synchrotron", Proceedings of the International Conference on High Energy Accelerators, 1967, MIT and Harvard University, pp. A91-A93.

[2] T. Bertuccio, M. Isaila, J. Kirchgessner, K. Koepke, F. larsen, A. Passner, K. Prelec, "Improvement in the RF Capture by Using Nonsinusoidal Accelerating Voltages", Proceedings of 1969 Particle Accelerator Conference, pp. 533-534.

[3] P. Bramham, S. Hansen, A. Hofmann, E. Peschardt, "Longitudinal Instabilities of Bunched Beams in the ISR", IEEE Trans. on Nucl. Science, Vol. NS-24, No. 3, 1977, pp. 1490-1492.

[4] A. Hofmann and S. Myers, "Beam Dynamics in a Double RF System", Proceedings of the 11th International Conference on High-Energy Accelerators, Geneva, 1980, pp. 610-614.

[5] M.Q. Barton, "Coalescence of Bunches in a Synchrotron to Change Acceleration Harmonic Number", BNL Report, BNL 33126, May 10, 1983, pp. 1-7.

[6] J.M. Ballod, L. Magnani, G. Nassibian, F. Pedersen, and W. Weisflog, "A Second Harmonic (6-16 MHz) RF System with Feedback-Reduced Gap Impedance for Accelerating Flat-Topped Bunches in the CERN PS Booster", IEEE Trans. on Nucl. Science, Vol. NS-30, No. 4, 1983, pp. 3499-3501.

[7] E.P. Colton, "Longitudinal Tune Control in Synchrotrons", 1984, pp. 437-443.

[8] J. Kats, "Synchronous Particle and Bucket Dynamics", BNL Report No. BNL-52171, October 3, 1988.

[9] A. Hofmann and F. Pedersen, "Bunches with Local Elliptic Energy Distribution" CERN/ISR-TH/79-17, PS/BR/79-11, 2 March 1979. 